Manuscript: cm0513177

\title{
Design of High Coordination Number Metallomesogens by Decoupling of the Complex-Forming and Mesogenic Groups: \\ Nematic and Lamello-Columnar Mesophases
}

Thomas Cardinaels, Kris Driesen, Tatjana N. Parac-Vogt, Benoît Heinrich, Cyril Bourgogne, Daniel Guillon, Bertrand Donnio, Koen Binnemans*

Supporting Information 


\section{General experimental information}

The nuclear magnetic resonance (NMR) spectra were recorded on a Bruker Avance 300 spectrometer (operating at $300 \mathrm{MHz}$ ) or a Bruker AMX-400 (operating at $400 \mathrm{MHz}$ ). FTIR spectra were recorded on a Bruker IFS-66 spectrometer, using the $\mathrm{KBr}$ pellet method. Elemental analyses were obtained on a CE-Instrument EA-1110 elemental analyzer. Optical textures of the mesophases were observed with an Olympus BX60 polarizing microscope equipped with a LINKAM THMS600 hot stage and a LINKAM TMS93 programmable temperature-controller. DSC traces were recorded with a Mettler-Toledo DSC821e module. The XRD patterns were obtained with two different experimental set-ups, and in all cases, the powdered sample was filled in Lindemann capillaries of $1 \mathrm{~mm}$ diameter. A linear monochromatic $\mathrm{CuK} \alpha_{1}$ beam $(\lambda=1.5405 \AA)$ obtained with a sealed-tube generator $(900 \mathrm{~W})$ and a bent quartz monochromator were used (both generator and monochromator were manufactured by Inel). One set of diffraction patterns was registered with a curved counter Inel CPS 120 , for which the sample temperature is controlled within $\pm 0.05^{\circ} \mathrm{C}$; periodicities up to $60 \AA$ can be measured. The other set of diffraction patterns was registered on Image Plate. Periodicities up to $90 \AA$ can be measured, and the sample temperature is controlled within $\pm 0.3{ }^{\circ} \mathrm{C}$. The molecular modeling calculations were performed on an SGI Origin 2800 20 CPU computer and on an SGI Octane ${ }^{2}$ workstation using the DISCOVER 3 molecular mechanics package from Accelrys (www.accelrys.com) with the esff force field. For all models, prior to the dynamics, the systems were minimized to a gradient of $0.1 \mathrm{kcal} \mathrm{mol}^{-1}$. The simulation then consisted of a 50 ps cycle from 373 to $353 \mathrm{~K}$, and a 200 ps isotherm at $353 \mathrm{~K}$ in the NVT-PBC ensemble and with a 1 fs time step.

Photoluminescence spectra have been recorded on an Edinburgh Instruments FS900 spectrofluorimeter. This instrument is equipped with a $450 \mathrm{~W}$ xenon arc lamp, a microsecond flashlamp and a red-sensitive photomultiplier $(250-850 \mathrm{~nm})$. All the photoluminescence 
spectra were recorded at room temperature and have been corrected for the sensitivity of the detector at the different wavelengths. The quantum yield of the europium sample was determined using an integrating sphere (150 mm diameter, $\mathrm{BaSO}_{4}$ coating) of Edinburgh Instruments. The quantum yield can be defined as the integrated intensity of luminescence signal divided by the integrated intensity of the absorption signal. Only the intense luminescence band around $612 \mathrm{~nm}$ of the europium(III) emission was measured by the integrating sphere, but this intensity value was corrected by taking into account the relative intensity of the other transitions (as determined from the steady state luminescence spectrum. The absorption intensity was obtained by subtracting the integrated intensity of the light source (with the europium sample in the integrating sphere) from the integrated intensity of the light source (with a blank sample in the integrating sphere). The blank sample used contained the solvent DMF. 


\section{$\underline{\text { Synthesis }}$}

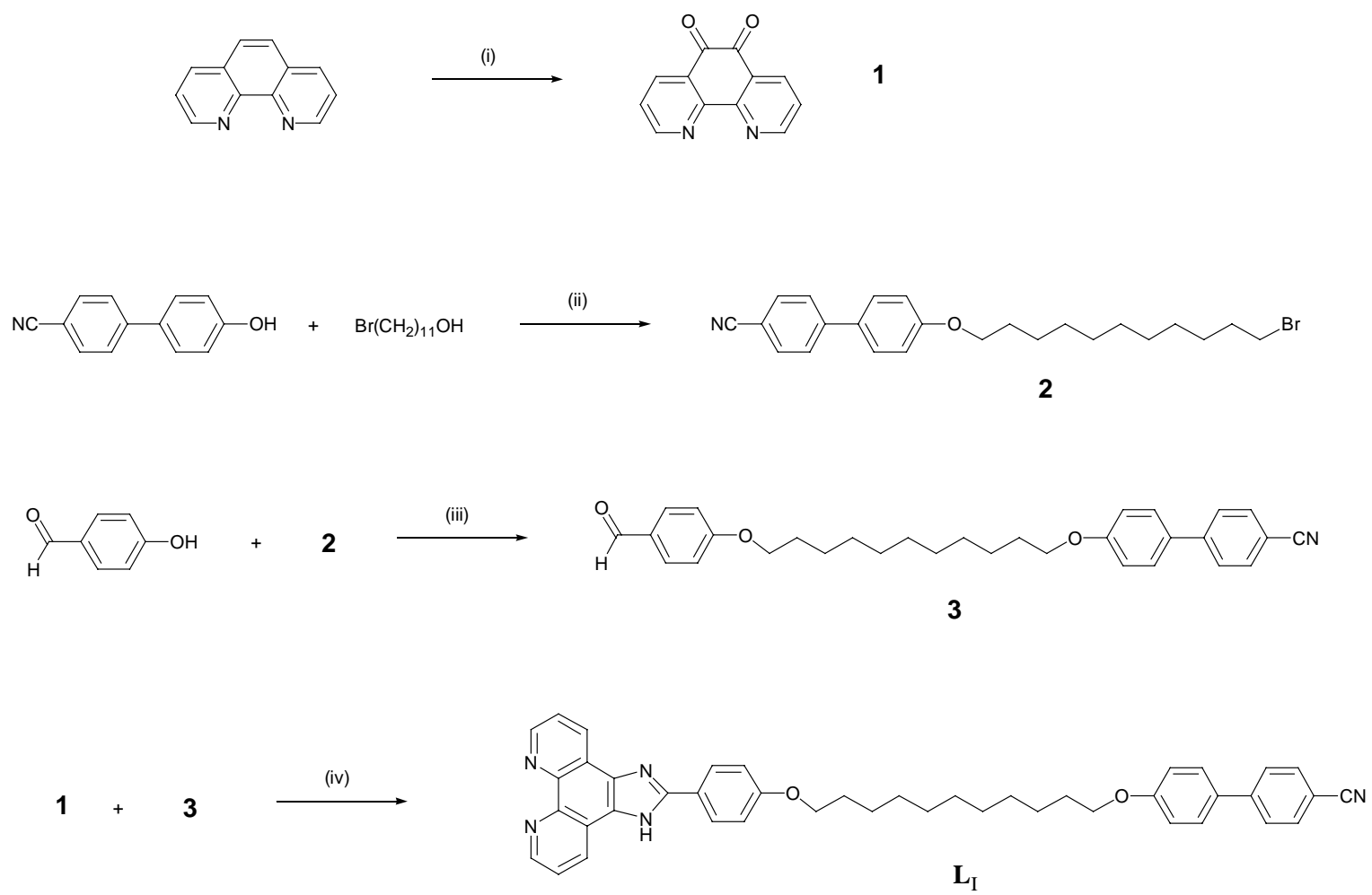

Scheme S1. Synthesis of ligand $\mathbf{L}_{\mathbf{I}}$. Experimental conditions: (i) $\mathrm{H}_{2} \mathrm{SO}_{4} / \mathrm{HNO}_{3}, \mathrm{NaBr}$; (ii) $\mathrm{PPh}_{3}$, DIAD, THF; (iii) $\mathrm{K}_{2} \mathrm{CO}_{3} / \mathrm{KI}$, 2-butanone; (iv) $\mathrm{NH}_{4} \mathrm{OAc}$, $\mathrm{HOAc}$.

The other ligands $\mathbf{L}_{\text {II }}$ and $\mathbf{L}_{\text {III }}$ being prepared by following a similar method starting from 3,4-dihydroxybenzaldehyde and from 3,4,5-trihydroxybenzaldehyde, respectively. 


\section{(a) 1,10-phenanthroline-5,6-dione (1)}

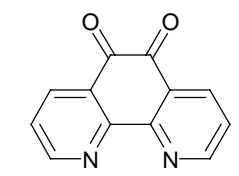

1,10-Phenanthroline monohydrate $(0.0504 \mathrm{~mol}, 10.00 \mathrm{~g})$ was added in small portions under stirring to $60 \mathrm{~mL}$ of concentrated sulfuric acid in a round-bottom flask $(500 \mathrm{~mL})$ equipped with a reflux condenser. After the solid compound was dissolved, sodium bromide $(0.0504$ mol, $5.19 \mathrm{~g}$ ) was added, followed by $30 \mathrm{~mL}$ of $70 \%$ nitric acid. The mixture was heated to $105^{\circ} \mathrm{C}$ for 6 hours. The temperature was then lowered to $95^{\circ} \mathrm{C}$ and the reflux condenser was removed to allow the bromine vapors to escape for a period of 16 hours. After being cooled, the mixture was poured onto $800 \mathrm{~g}$ of ice and was carefully neutralized to $\mathrm{pH} 7$ with about $300 \mathrm{~mL}$ of a $10 \mathrm{M}$ solution of sodium hydroxide. The turbid solution was filtered and the solid residue was extracted with $5 \times 200 \mathrm{~mL}$ of boiling water. The insoluble material was removed from the cooled extraction liquid by filtration and the combined aqueous solutions were extracted with dichloromethane, dried over anhydrous $\mathrm{MgSO}_{4}$, and the solvent was removed under reduced pressure. The crude product was recrystallized from toluene to

obtain orange crystals. Yield: $30 \%(3.18 \mathrm{~g}) .{ }^{1} \mathrm{H}$ NMR (300 MHz, $\left.\mathrm{CDCl}_{3}, \delta \mathrm{ppm}\right): 7.58-7.62$ $\left(\mathrm{dd}, 2 \mathrm{H}, \mathrm{H}\right.$-aryl, $\left.J_{o}=8.1 \mathrm{~Hz}, J_{o}=4.8 \mathrm{~Hz}\right), 8.53\left(\mathrm{dd}, 2 \mathrm{H}, \mathrm{H}-\operatorname{aryl}, J_{o}=8.1 \mathrm{~Hz}, J_{m}=1.8 \mathrm{~Hz}\right)$, $9.12\left(\mathrm{dd}, 2 \mathrm{H}, \mathrm{H}\right.$-aryl, $\left.J_{o}=4.8 \mathrm{~Hz}, J_{m}=1.8 \mathrm{~Hz}\right)$. Calcd. for $\left(\mathrm{C}_{12} \mathrm{H}_{6} \mathrm{~N}_{2} \mathrm{O}_{2}\right)_{3} \mathrm{H}_{2} \mathrm{O}: \mathrm{C} 66.67, \mathrm{H}$ 3.11, N 12.96. Found: C 67.25, H 2.77, N 12.99. M.p.: $257^{\circ} \mathrm{C}$. 


\section{(b) 4'-(10-Bromodecyloxy)-4-cyanobiphenyl (2)}

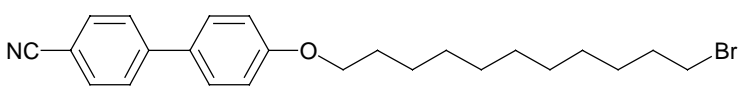

Bromide 2 was prepared by adding diisopropyl azodicarboxylate (DIAD) (0.0256 mol, 5.18 g) dropwise to an ice-cooled stirred solution of 4'-hydroxy-4-biphenylcarbonitrile $(0.0256$ mol, $5.00 \mathrm{~g}), 11$-bromo-1-undecanol (0.0256 mol, $6.43 \mathrm{~g})$ and triphenylphosphine $(0.0256$ mol, $6.72 \mathrm{~g}$ ) in dry THF, in a nitrogen atmosphere. The solution was allowed to warm up to room temperature and stirred for 24 hours. The solvent was removed under reduced pressure and the crude product was purified on a silica column with $\mathrm{CHCl}_{3}$ as the eluent. The product was recrystallized from hexane to obtain white crystals. Yield: 67\% (7.35 g). ${ }^{1} \mathrm{H}$ NMR (300 $\left.\mathrm{MHz}, \mathrm{CDCl}_{3}, \delta \mathrm{ppm}\right): 1.32-1.51\left(\mathrm{~m}, 14 \mathrm{H}, \mathrm{CH}_{2}\right), 1.82-1.92\left(\mathrm{~m}, 4 \mathrm{H},-\mathrm{CH}_{2}-\mathrm{CH}_{2}-\mathrm{Br}\right.$ and $-\mathrm{CH}_{2}-\mathrm{CH}_{2}-\mathrm{O}-$ ), 3.42 (t, 2H, $\left.-\mathrm{CH}_{2}-\mathrm{Br}\right), 4.02$ (t, 2H, $-\mathrm{CH}_{2}-\mathrm{O}-$ ), 7.02 (d, 2H, H-aryl, $J_{o}=$ $8.8 \mathrm{~Hz}), 7.52\left(\mathrm{~d}, 2 \mathrm{H}, \mathrm{H}\right.$-aryl, $\left.J_{o}=8.8 \mathrm{~Hz}\right), 7.63-7.72(\mathrm{~m}, 4 \mathrm{H}, \mathrm{H}-\operatorname{aryl}) .{ }^{13} \mathrm{C} \mathrm{NMR}(300 \mathrm{MHz}$, $\mathrm{CDCl}_{3}, \delta$ ppm): 26.43, 28.56, 29.15, 29.63, 29.75, 29.81, 29.85, 29.92, 33.24, 34.45, 68.57, 110.44, 115.50, 119.52, 127.47, 128.72, 131.64, 132.97, 145.69, 160.22. IR (KBr-pellet, $\mathrm{cm}^{-}$ $\left.{ }^{1}\right): 2918,2850$ (s, aliphatic C-H stretch), $2222\left(\mathrm{~m}, \mathrm{v}(\mathrm{C} \equiv \mathrm{N})\right.$ ). Calcd. for $\mathrm{C}_{24} \mathrm{H}_{30} \mathrm{BrNO}$ : C 67.29, H 7.06, N 3.27. Found: C 67.32, H 7.00, N 3.35. EI (m/z): 427.2, M $^{+}$. M.p.: $77^{\circ} \mathrm{C}(\mathrm{a}$ monotropic nematic phase is formed upon cooling at $66^{\circ} \mathrm{C}$ ). 


\section{(c) Aldehyde 3}

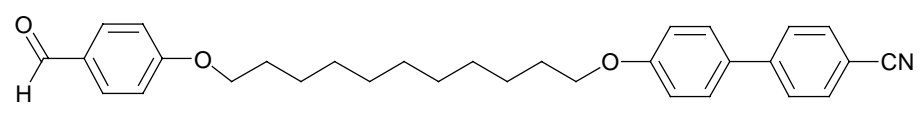

Aldehyde 3 was prepared by adding bromide $2(0.0070 \mathrm{~mol}, 3.00 \mathrm{~g})$ to a solution of $4-$ hydroxybenzaldehyde $(0.0070 \mathrm{~mol}, 0.85 \mathrm{~g})$ and potassium carbonate $(0.0070 \mathrm{~mol}, 0.97 \mathrm{~g})$ in 2-butanone $(50 \mathrm{~mL})$. A catalytic amount of potassium iodide was added and the mixture was refluxed for 24 hours protected by a guard tube. After the reaction mixture was cooled to room temperature, it was poured into $200 \mathrm{~mL}$ of water. The organic layer was separated and the aqueous layer was extracted with dichloromethane. The combined organic layers were dried over anhydrous $\mathrm{MgSO}_{4}$ and the solvent was removed under reduced pressure. The crude product was recrystallized from ethyl acetate. Yield: 70\% (2.30 g). ${ }^{1} \mathrm{H}$ NMR (300 $\left.\mathrm{MHz}, \mathrm{CDCl}_{3}, \delta \mathrm{ppm}\right): 1.34-1.49\left(\mathrm{~m}, 14 \mathrm{H}, \mathrm{CH}_{2}\right), 1.80-1.87\left(\mathrm{~m}, 4 \mathrm{H},-\mathrm{CH}_{2}-\mathrm{CH}_{2}-\mathrm{O}-\right)$, 4.00-4.07 (m, 4H, -CH $\mathbf{H}_{2}-\mathrm{O}-$ ), 6.99-7.02 (m, 4H, H-aryl), 7.52 (d, 2H, H-aryl, $\left.J_{o}=8.8 \mathrm{~Hz}\right)$, 7.63-7.72 (m, 4H, H-aryl), 7.83 (d, 2H, H-aryl, $\left.J_{o}=8.1 \mathrm{~Hz}\right), 9.89(\mathrm{~s}, 1 \mathrm{H}, \mathrm{CH}=\mathrm{O}) .{ }^{13} \mathrm{C} \mathrm{NMR}$ (300 MHz, $\mathrm{CDCl}_{3}, \delta$ ppm): 26.37, 26.44, 29.46, 29.63, 29.73, 29.78, 29.93, 68.56, 68.82, $110.44,115.15,115.47,119.53,127.47,128.72,130.15,131.66,132.40,132.97,145.67$, 160.20, 164.65, 191.23. IR (KBr-pellet, $\mathrm{cm}^{-1}$ ): 2922, 2852 (s, aliphatic C-H stretch), 2222 $(\mathrm{m}, \mathrm{v}(\mathrm{C} \equiv \mathrm{N})), 1689(\mathrm{~s}, \mathrm{v}(\mathrm{C}=\mathrm{O}))$. Calcd. for $\left(\mathrm{C}_{31} \mathrm{H}_{35} \mathrm{NO}_{3}\right)_{3} \mathrm{H}_{2} \mathrm{O}: \mathrm{C} 78.28, \mathrm{H}$ 7.56, N 2.94. Found: C 78.38, H 7.08, N 2.97. ESI-MS (Acetonitrile, m/z): 470.3, $[\mathrm{M}+\mathrm{H}]^{+}$. M.p.: $95{ }^{\circ} \mathrm{C}$ (a monotropic nematic phase is formed upon cooling at $89^{\circ} \mathrm{C}$ ). 


\section{(d) Aldehyde 4}

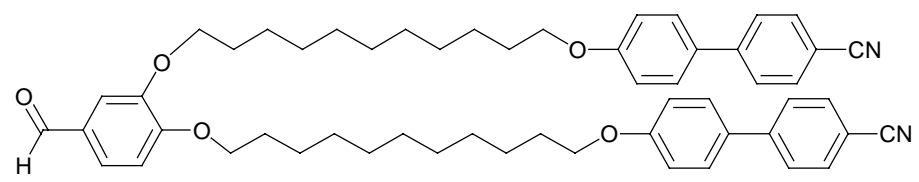

Aldehyde 4 was prepared by adding bromide $2(0.0145 \mathrm{~mol}, 6.20 \mathrm{~g})$ to a solution of 3,4dihydroxybenzaldehyde $(0.0072 \mathrm{~mol}, 1.00 \mathrm{~g})$ and potassium carbonate $(0.0145 \mathrm{~mol}, 2.00 \mathrm{~g})$ in 2-butanone $(100 \mathrm{~mL})$. A catalytic amount of potassium iodide was added and the mixture was refluxed for 24 hours protected by a guard tube. After the reaction mixture was cooled to room temperature, it was poured into $200 \mathrm{~mL}$ of water. The organic layer was separated and the aqueous layer was extracted with dichloromethane. The combined organic layers were dried over anhydrous $\mathrm{MgSO}_{4}$ and the solvent was removed under reduced pressure. The crude product was recrystallized from ethylacetate. Yield: $60 \%$ (3.62 g). ${ }^{1} \mathrm{H}$ NMR (300 $\left.\mathrm{MHz}, \mathrm{CDCl}_{3}, \delta \mathrm{ppm}\right): 1.34-1.49\left(\mathrm{~m}, 28 \mathrm{H}, \mathrm{CH}_{2}\right), 1.77-1.90\left(\mathrm{~m}, 8 \mathrm{H},-\mathrm{CH}_{2}-\mathrm{CH}_{2}-\mathrm{O}-\right)$, 3.99-4.11 (m, 8H, -CH $\mathbf{C H}_{2}-\mathrm{O}-$ ), 6.95-7.01 (m, 5H, H-aryl), 7.41-7.44 (m, 2H, H-aryl), 7.52 (d, 4H, H-aryl, $\left.J_{o}=8.0 \mathrm{~Hz}\right), 7.62-7.70\left(\mathrm{~m}, 8 \mathrm{H}, \mathrm{H}\right.$-aryl), $9.84(\mathrm{~s}, 1 \mathrm{H}, \mathrm{CH}=\mathrm{O}) .{ }^{13} \mathrm{C} \mathrm{NMR}(300$ $\left.\mathrm{MHz}, \mathrm{CDCl}_{3}, \delta \mathrm{ppm}\right): 26.37,26.46,29.38,29.46,29.64,29.75,29.81,29.93,29.98,68.56$, $69.50,110.46,111.29,112.18,115.48,119.50,127.06,127.45,128.70,130.29,131.64$, 132.95, 145.66, 149.83, 155.05, 160.20, 191.39. IR (KBr-pellet, $\left.\mathrm{cm}^{-1}\right): 2919,2849$ (s, aliphatic $\mathrm{C}-\mathrm{H}$ stretch), $2224(\mathrm{~m}, v(\mathrm{C} \equiv \mathrm{N})), 1685(\mathrm{~s}, \mathrm{v}(\mathrm{C}=\mathrm{O}))$. Calcd. for $\mathrm{C}_{55} \mathrm{H}_{64} \mathrm{~N}_{2} \mathrm{O}_{5}: \mathrm{C}$ 79.29, H 7.74, N 3.36. Found: C 78.98, H 8.08, N 3.45. ESI-MS (Acetonitrile, m/z): 833.3, $[\mathrm{M}+\mathrm{H}]^{+}$. The compound is an enantiotropic liquid crystal: $\mathrm{Cr} \cdot 54 \cdot \mathrm{N} \cdot 89 \cdot \mathrm{I}$. 


\section{(e) Aldehyde 5}

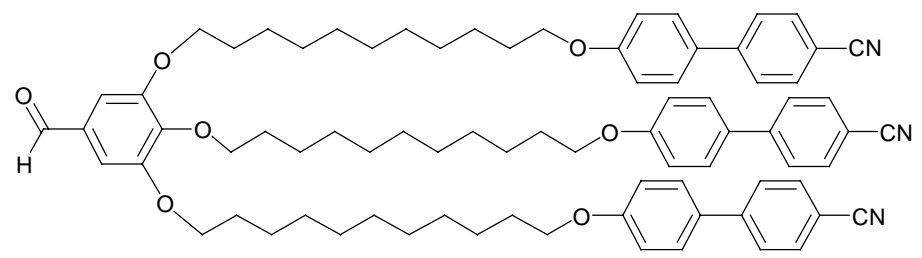

Aldehyde 5 was prepared by adding bromide $2(0.0070 \mathrm{~mol}, 3.00 \mathrm{~g})$ to a solution of 3,4,5trihydroxybenzaldehyde monohydrate $(0.0023 \mathrm{~mol}, 0.40 \mathrm{~g})$ and potassium carbonate $(0.0070$ mol, $0.97 \mathrm{~g})$ in 2-butanone $(50 \mathrm{~mL})$. A catalytic amount of potassium iodide was added and the mixture was refluxed for 24 hours protected by a guard tube. After the reaction mixture was cooled to room temperature, it was poured into $200 \mathrm{~mL}$ of water. The organic layer was separated and the aqueous layer was extracted with dichloromethane. The combined organic layers were dried over anhydrous $\mathrm{MgSO}_{4}$ and the solvent was removed under reduced pressure. The crude product was recrystallized from toluene. Yield: 47\% (1.30 g). ${ }^{1} \mathrm{H}$ NMR (300 MHz, $\left.\mathrm{CDCl}_{3}, \delta \mathrm{ppm}\right): 1.34-1.49\left(\mathrm{~m}, 42 \mathrm{H}, \mathrm{CH}_{2}\right), 1.77-1.87\left(\mathrm{~m}, 12 \mathrm{H},-\mathrm{CH}_{2}-\mathrm{CH}_{2}-\mathrm{O}-\right)$, 3.98-4.10 (m, 12H, - $\left.\mathrm{CH}_{2}-\mathrm{O}-\right), 7.01\left(\mathrm{dd}, 6 \mathrm{H}, \mathrm{H}-\operatorname{aryl}, J_{o}=8.8 \mathrm{~Hz}, J_{m}=2.5 \mathrm{~Hz}\right), 7.10(\mathrm{~s}, 2 \mathrm{H}$, H-aryl), $7.52\left(\mathrm{dd}, 6 \mathrm{H}, \mathrm{H}\right.$-aryl, $\left.J_{o}=8.7 \mathrm{~Hz}, J_{m}=1.9 \mathrm{~Hz}\right), 7.63-7.71(\mathrm{~m}, 12 \mathrm{H}, \mathrm{H}-\operatorname{aryl}), 9.84(\mathrm{~s}$, $1 \mathrm{H}, \mathrm{CH}=\mathrm{O}) .{ }^{13} \mathrm{C} \mathrm{NMR}\left(300 \mathrm{MHz}, \mathrm{CDCl}_{3}, \delta \mathrm{ppm}\right): 26.44,29.64,29.76,29.81,29.95,29.98$, $30.04,30.75,68.56,69.62,74.01,108.26,110.46,115.48,119.49,127.44,128.70,131.66$, 131.87, 132.95, 144.22, 145.64, 153.91, 160.20, 191.65. IR (KBr-pellet, $\left.\mathrm{cm}^{-1}\right): 2922,2850$ (s, aliphatic C-H stretch), $2222(\mathrm{~m}, \mathrm{v}(\mathrm{C} \equiv \mathrm{N})), 1693(\mathrm{~s}, \mathrm{v}(\mathrm{C}=\mathrm{O}))$. Calcd. for $\mathrm{C}_{79} \mathrm{H}_{93} \mathrm{~N}_{3} \mathrm{O}_{7}$ : $\mathrm{C}$ 79.30, H 7.83, N 3.51. Found: C 79.10, H 7.66, N 3.34. ESI-MS (MeOH, m/z): 1218.7, [M + $\mathrm{Na}]^{+}$. The compound is an enantiotropic liquid crystal: $\mathrm{Cr} \cdot 104 \cdot \mathrm{N} \cdot 111 \cdot \mathrm{I}$. 


\section{(f) ligand $\mathbf{L}_{\mathbf{I}}$}

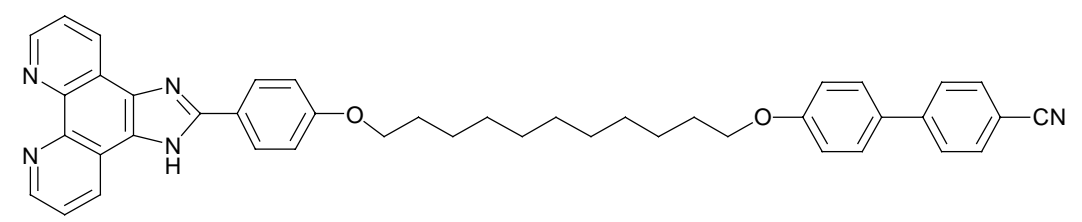

Ligand $\mathbf{L}_{\mathbf{I}}$ was prepared by adding aldehyde $3(0.0021 \mathrm{~mol}, 1.00 \mathrm{~g})$ to a warm solution of 1,10-phenanthroline-5,6-dione $(0.0021 \mathrm{~mol}, 0.45 \mathrm{~g})$ and ammonium acetate $(0.0085 \mathrm{~mol}$, $0.66 \mathrm{~g}$ ) in $30 \mathrm{~mL}$ of glacial acetic acid. The mixture was heated to $90{ }^{\circ} \mathrm{C}$ for 3 hours. After the reaction mixture was cooled to room temperature, it was poured into $100 \mathrm{~mL}$ of water and neutralized to $\mathrm{pH} 7$ with an aqueous ammonia solution. The precipitate was filtered, washed with distilled water and dried. The crude product was purified on a silica column with $\mathrm{CHCl}_{3} / \mathrm{MeOH}(90: 10)$ as the eluent. Since the compound holds solvents firmly, it was dried in a vacuum oven at $60{ }^{\circ} \mathrm{C}$. Yield: $36 \%(0.50 \mathrm{~g}) .{ }^{1} \mathrm{H}$ NMR $(400 \mathrm{MHz}, \mathrm{DMSO}, 333 \mathrm{~K}, \delta$ ppm): 1.31-1.50 (m, 14H, $\left.\mathrm{CH}_{2}\right), 1.69-1.80\left(\mathrm{~m}, 4 \mathrm{H},-\mathrm{CH}_{2}-\mathrm{CH}_{2}-\mathrm{O}-\right), 4.01(\mathrm{t}, 2 \mathrm{H}$, $-\mathrm{CH}_{2}-\mathrm{O}-$ ), 4.09 (t, 2H, -CH $\left.\mathbf{H}_{2}-\mathrm{O}-\right), 7.02$ (d, 2H, H-aryl, $\left.J_{o}=8.8 \mathrm{~Hz}\right), 7.15$ (d, 2H, H-aryl, $J_{o}$ $=8.8 \mathrm{~Hz}), 7.62\left(\mathrm{~d}, 2 \mathrm{H}, \mathrm{H}-\operatorname{aryl}, J_{o}=8.8 \mathrm{~Hz}\right), 7.76-7.81(\mathrm{~m}, 6 \mathrm{H}, \mathrm{H}$-aryl $), 8.20$ (d, 2H, H-aryl, $\left.J_{o}=8.9 \mathrm{~Hz}\right), 8.90\left(\mathrm{~d}, 2 \mathrm{H}, \mathrm{H}-\operatorname{aryl}, J_{o}=7.3 \mathrm{~Hz}\right), 9.03\left(\mathrm{~d}, 2 \mathrm{H}, \mathrm{H}-\operatorname{aryl}, J_{o}=4.2 \mathrm{~Hz}\right), 13.40(\mathrm{~s}, 1 \mathrm{H}$, $\mathrm{N}-\mathrm{H}) .{ }^{13} \mathrm{C}$ NMR (400 MHz, DMSO, 333K, $\left.\delta \mathrm{ppm}\right): 25.44,28.66,28.78,28.84,28.95,67.87$, $67.95,109.25,115.06,115.28,118.81,122.75,123.07,126.81,127.90,128.20,129.40$, 130.45, 132.62, 143.66, 144.39, 147.54, 150.90, 159.49, 160.08. IR (KBr-pellet, $\left.\mathrm{cm}^{-1}\right): 3404$ (m, v(N-H)), 2920, 2850 (s, aliphatic C-H stretch), $2224(\mathrm{~m}, \mathrm{v}(\mathrm{C} \equiv \mathrm{N}))$. Calcd. for $\left(\mathrm{C}_{43} \mathrm{H}_{41} \mathrm{~N}_{5} \mathrm{O}_{2}\right)\left(\mathrm{H}_{2} \mathrm{O}\right): \mathrm{C} 76.19, \mathrm{H} 6.39, \mathrm{~N}$ 10.33. Found: C 76.51, H 6.22, N 9.87. ESI-MS (Acetonitrile, $\mathrm{m} / \mathrm{z}$ ): $660.5,[\mathrm{M}+\mathrm{H}]^{+}$. The compound is an enantiotropic liquid crystal: $\mathrm{Cr}$. $80 \cdot \mathrm{N} \cdot 124 \cdot \mathrm{I}$ 


\section{(g) Ligand $\mathbf{L}_{\text {II }}$}

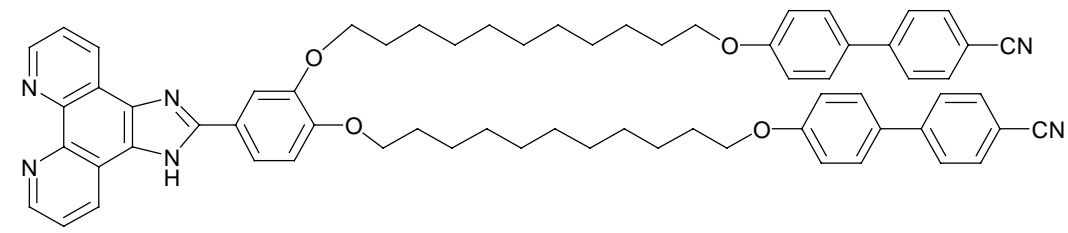

Ligand $\mathbf{L}_{\text {II }}$ was prepared by adding aldehyde $4(0.0024 \mathrm{~mol}, 2.00 \mathrm{~g})$ to a warm solution of 1,10-phenanthroline-5,6-dione $(0.0024 \mathrm{~mol}, 0.50 \mathrm{~g})$ and ammonium acetate $(0.0096 \mathrm{~mol}$, $0.74 \mathrm{~g}$ ) in $30 \mathrm{~mL}$ of glacial acetic acid. The mixture was heated to $90{ }^{\circ} \mathrm{C}$ for 3 hours. After the reaction mixture was cooled to room temperature, it was poured into $100 \mathrm{~mL}$ of water and neutralized to $\mathrm{pH} 7$ with an aqueous ammonia solution. The precipitate was filtered, washed with distilled water and dried. The crude product was purified on a silica column with $\mathrm{CHCl}_{3} / \mathrm{MeOH}(90: 10)$ as the eluent. Since the compound holds solvents firmly, it was dried in a vacuum oven at $60{ }^{\circ} \mathrm{C}$. Yield: $35 \%(0.86 \mathrm{~g}) .{ }^{1} \mathrm{H}$ NMR $(400 \mathrm{MHz}, \mathrm{DMSO}, 333 \mathrm{~K}, \delta$ ppm): 1.27-1.47 (m, 28H, $\left.\mathrm{CH}_{2}\right), 1.64-1.76\left(\mathrm{~m}, 8 \mathrm{H},-\mathrm{CH}_{2}-\mathrm{CH}_{2}-\mathrm{O}-\right), 3.92-3.96(\mathrm{~m}, 4 \mathrm{H}$, $\left.-\mathrm{CH}_{2}-\mathrm{O}-\right), 4.03-4.10\left(\mathrm{~m}, 4 \mathrm{H},-\mathrm{CH}_{2}-\mathrm{O}-\right), 6.96\left(\mathrm{dd}, 4 \mathrm{H}, \mathrm{H}-\operatorname{aryl}, J_{o}=8.8 \mathrm{~Hz}, J_{m}=2.4 \mathrm{~Hz}\right)$, $7.13\left(\mathrm{~d}, 1 \mathrm{H}, \mathrm{H}-\operatorname{aryl}, J_{o}=8.2 \mathrm{~Hz}\right), 7.56\left(\mathrm{~d}, 4 \mathrm{H}, \mathrm{H}-\operatorname{aryl}, J_{o}=8.8 \mathrm{~Hz}\right), 7.72\left(\mathrm{~d}, 4 \mathrm{H}, \mathrm{H}-\operatorname{aryl}, J_{o}=\right.$ 8.5 Hz), 7.75-7.79 (m, 6H, H-aryl), 7.87 (m, 2H, H-aryl), $8.91\left(\mathrm{~d}, 2 \mathrm{H}, \mathrm{H}\right.$-aryl, $\left.J_{o}=8.1 \mathrm{~Hz}\right)$, $9.01\left(\mathrm{~d}, 2 \mathrm{H}, \mathrm{H}-\operatorname{aryl}, J_{o}=4.1 \mathrm{~Hz}\right), 13.55(\mathrm{~s}, 1 \mathrm{H}, \mathrm{N}-\mathrm{H}) .{ }^{13} \mathrm{C} \mathrm{NMR}(400 \mathrm{MHz}, \mathrm{DMSO}, 333 \mathrm{~K}$, $\delta$ ppm): 25.06, 25.15, 25.17, 28.29, 28.32, 28.35, 28.43, 28.45, 28.51, 28.56, 28.63, 67.42, $68.50,68.97,108.84,112.65,114.02,114.78,118.37,119.49,122.65,122.80,126.33$, $127.72,129.23,129.99,132.17,142.99,143.92,147.02,148.74,150.20,150.56,159.05$. IR (KBr-pellet, $\left.\mathrm{cm}^{-1}\right): 3435(\mathrm{~s}, \mathrm{v}(\mathrm{N}-\mathrm{H})), 2924,2853$ (s, aliphatic C-H stretch), 2224 (m, $v(\mathrm{C} \equiv \mathrm{N}))$. Calcd. for $\left(\mathrm{C}_{67} \mathrm{H}_{70} \mathrm{~N}_{6} \mathrm{O}_{4}\right)\left(\mathrm{H}_{2} \mathrm{O}\right)$ : C 77.28, H 6.97, N 8.07. Found: C 77.76, H 6.99, N 8.11. ESI-MS (Acetonitrile, $\mathrm{m} / \mathrm{z}$ ): 1023.5, $[\mathrm{M}+\mathrm{H}]^{+}$. The compound is an enantiotropic liquid crystal: $\mathrm{Cr} \cdot 75 \cdot \mathrm{N} \cdot 128 \cdot \mathrm{I}$. 


\section{(h) Ligand $\mathrm{L}_{\text {III }}$}

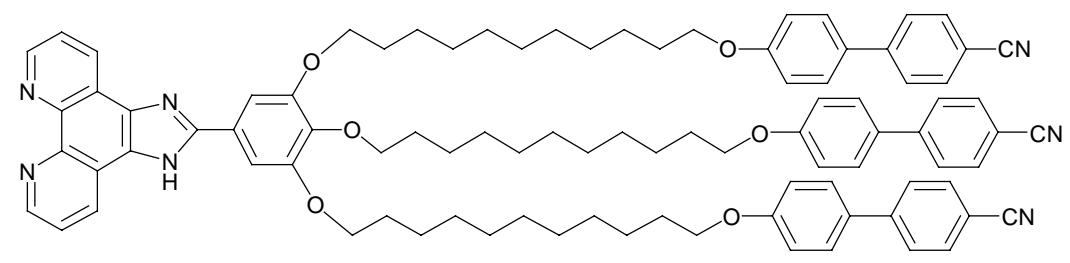

Ligand $\mathbf{L}_{\text {III }}$ was prepared by adding aldehyde $5(0.58 \mathrm{mmol}, 0.700 \mathrm{~g})$ to a warm solution of 1,10-phenanthroline-5,6-dione (0.58 mmol, $0.123 \mathrm{~g})$ and ammonium acetate $(5.8 \mathrm{mmol}$, $0.450 \mathrm{~g}$ ) in $30 \mathrm{~mL}$ of glacial acetic acid. The mixture was heated to $90{ }^{\circ} \mathrm{C}$ for 3 hours. After the reaction mixture was cooled to room temperature, it was poured into $100 \mathrm{~mL}$ of water and neutralized to $\mathrm{pH} 7$ with an aqueous ammonia solution. The precipitate was filtered, washed with distilled water and dried. The crude product was purified on a silica column with $\mathrm{CHCl}_{3} / \mathrm{MeOH}(90: 10)$ as the eluent. Since the compound holds solvents firmly, it was dried in a vacuum oven at $60{ }^{\circ} \mathrm{C}$. Yield: $25 \%$ (0.200 g). ${ }^{1} \mathrm{H}$ NMR (300 MHz, DMSO, 343K, $\delta$ ppm): $1.27-1.52\left(\mathrm{~m}, 42 \mathrm{H}, \mathrm{CH}_{2}\right), 1.69-1.83\left(\mathrm{~m}, 12 \mathrm{H},-\mathrm{CH}_{2}-\mathrm{CH}_{2}-\mathrm{O}-\right), 3.97-3.99(\mathrm{~m}, 8 \mathrm{H}$, $\left.-\mathrm{CH}_{2}-\mathrm{O}-\right), 4.15$ (t, 4H, -CH $2-\mathrm{O}-$ ), 7.00 (d, 6H, H-aryl), 7.59-7.62 (m, 8H, H-aryl), 7.76 (m, 14H, H-aryl), 8.90 (d, 2H, H-aryl), 9.02 (d, 2H, H-aryl), 13.37 (s, 1H, N-H). ${ }^{13} \mathrm{C}$ NMR (400 MHz, DMSO, 333K, $\delta$ ppm): 25.43, 25.59, 28.68, 28.71, 28.81, 28.89, 28.91, 28.98, $29.02,29.85,67.82,69.16,72.74,105.81,109.23,115.19,118.77,122.98,125.19,126.74$, $128.13,128.84,129.47,130.41,132.58,139.58,143.76,144.33,147.65,150.75,153.09$, 159.45. IR (KBr-pellet, $\left.\mathrm{cm}^{-1}\right): 3446(\mathrm{~m}, \mathrm{v}(\mathrm{N}-\mathrm{H})), 2924,2852$ (s, aliphatic C-H stretch), $2224(\mathrm{~m}, \mathrm{v}(\mathrm{C} \equiv \mathrm{N}))$. Calcd. for $\left(\mathrm{C}_{91} \mathrm{H}_{99} \mathrm{~N}_{7} \mathrm{O}_{6}\right)\left(\mathrm{H}_{2} \mathrm{O}\right): \mathrm{C} 77.80, \mathrm{H}$ 7.25, N 6.98. Found: $\mathrm{C} 77.38$, H 7.21, N 6.74. ESI-MS (Acetonitrile, $\mathrm{m} / \mathrm{z}$ ): 1386.7, $[\mathrm{M}+\mathrm{H}]^{+}$. The compound is an enantiotropic liquid crystal: $\mathrm{Cr} \cdot 48 \cdot \mathrm{SmA}_{1 \mathrm{~d}} \cdot 94 \cdot \mathrm{I}$. 


\section{(i) Rhenium complex $\left[\operatorname{Re}(\mathrm{CO})_{3} \mathrm{BrL}_{\mathrm{I}}\right]$}

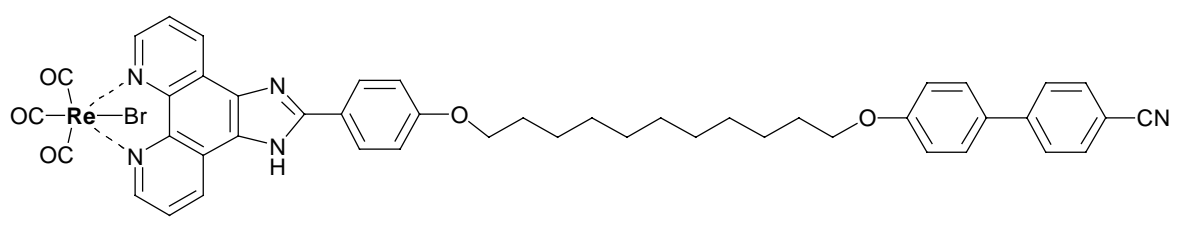

Rhenium(I) complex $\left[\operatorname{Re}(\mathrm{CO})_{3} \mathrm{BrL}_{\mathbf{I}}\right]$ was prepared by adding rhenium(I) pentacarbonyl bromide, $\operatorname{Re}(\mathrm{CO})_{5} \operatorname{Br}(0.17 \mathrm{mmol}, 0.068 \mathrm{~g})$ to a solution of ligand $\mathbf{L}_{\mathbf{I}}(0.17 \mathrm{mmol}, 0.110 \mathrm{~g})$ in toluene. The mixture was refluxed for 3 hours. Then the solvent was removed under reduced pressure and the crude product was purified on a silica column with $\mathrm{CHCl}_{3} / \mathrm{MeOH}(95: 5)$ as the eluent. The complex was dissolved in a minimum amount of $\mathrm{CHCl}_{3}$ and precipitated in hexane. The yellow precipitate was filtered off and dried in a vacuum oven at $60{ }^{\circ} \mathrm{C}$. Yield: 45\% (0.077 g). IR (KBr-pellet, $\left.\mathrm{cm}^{-1}\right): 3404(\mathrm{~m}, \mathrm{v}(\mathrm{N}-\mathrm{H})), 2926,2852$ (s, aliphatic C-H stretch), $2226(\mathrm{~m}, v(\mathrm{C} \equiv \mathrm{N}))$. Calcd. for $\left(\mathrm{C}_{46} \mathrm{H}_{41} \mathrm{BrN}_{5} \mathrm{O}_{5} \mathrm{Re}\right)\left(\mathrm{H}_{2} \mathrm{O}\right)$ : C 53.75, H 4.22, N 6.81. Found: C 53.87, H 4.20, N 6.63. ESI-MS (Acetonitrile, m/z): 1008.1, [M - H] $]^{-}$ 


\section{(j) Neodymium complex $\left[\mathrm{Nd}(\mathrm{tta})_{3} \mathrm{~L}_{\mathrm{I}}\right]$}

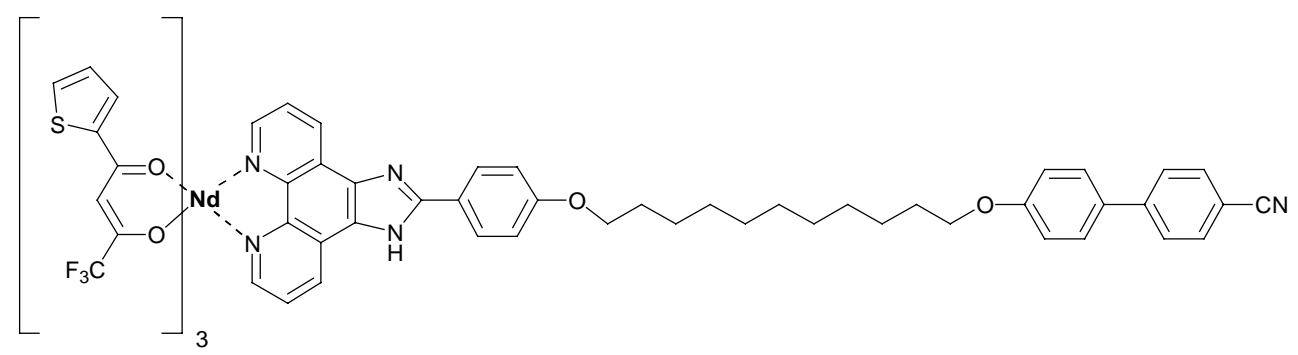

Neodymium(III) complex $\left[\mathrm{Nd}(\mathrm{tta})_{3} \mathbf{L}_{\mathbf{I}}\right]$ was prepared by adding a solution of $\mathrm{Nd}(\mathrm{tta})_{3} \cdot 2 \mathrm{H}_{2} \mathrm{O}$ (0.076 mmol, $0.064 \mathrm{~g})$ in $\mathrm{CHCl}_{3}$ to a solution of ligand $\mathbf{L}_{\mathbf{I}}(0.076 \mathrm{mmol}, 0.050 \mathrm{~g})$ in $\mathrm{CHCl}_{3}$. The mixture was refluxed for 1 hour. Then the solvent was removed under reduced pressure. The product was dissolved in a minimum amount of $\mathrm{CHCl}_{3}$ and precitated in hexane. The yellow precipitate was filtered off and dried in a vacuum oven at $60{ }^{\circ} \mathrm{C}$. Yield: $80 \%(0.089$

g). IR (KBr-pellet, $\left.\mathrm{cm}^{-1}\right): 3406(\mathrm{~m}, \mathrm{v}(\mathrm{N}-\mathrm{H})), 2928,2854$ (s, aliphatic C-H stretch), 2226 (m, $v(\mathrm{C} \equiv \mathrm{N}))$. Calcd. for $\left(\mathrm{C}_{67} \mathrm{H}_{53} \mathrm{~F}_{9} \mathrm{~N}_{5} \mathrm{NdO}_{8} \mathrm{~S}_{3}\right)\left(\mathrm{H}_{2} \mathrm{O}\right): \mathrm{C} 54.17, \mathrm{H} 3.73, \mathrm{~N}$ 4.71. Found: $\mathrm{C} 54.15, \mathrm{H}$ 3.76, N 4.84. ESI-MS (Acetonitrile, m/z): 1466.9, [M - H] $]^{-}$. 


\section{(k) Europium complex $\left[\mathrm{Eu}(\mathrm{tta})_{3} \mathbf{L}_{\mathrm{I}}\right]$}

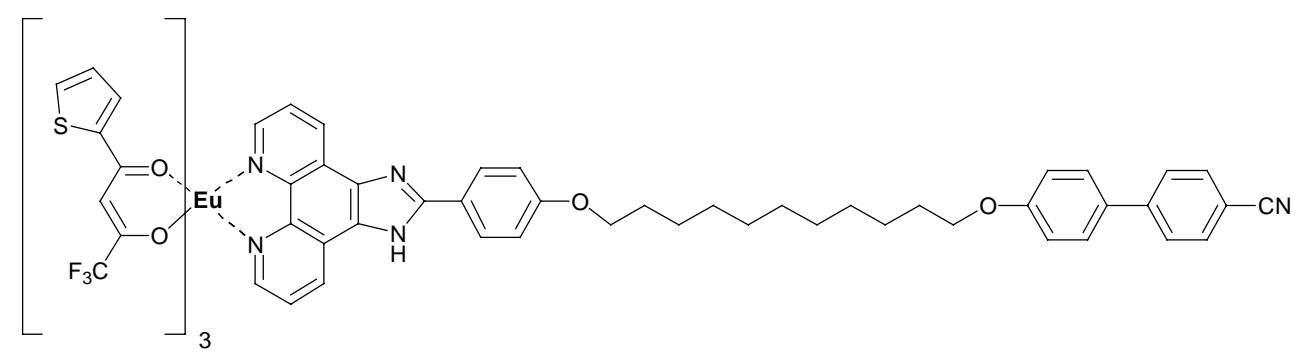

Europium(III) complex $\left[\mathrm{Eu}(\mathrm{tta})_{3} \mathbf{L}_{\mathbf{I}}\right]$ was prepared starting from ligand $\mathbf{L}_{\mathbf{I}}(0.076 \mathrm{mmol}$,

$0.050 \mathrm{~g})$ and $\mathrm{Eu}(\mathrm{tta})_{3} \cdot 2 \mathrm{H}_{2} \mathrm{O}(0.076 \mathrm{mmol}, 0.065 \mathrm{~g})$ using the procedure described for the $\mathrm{Nd}-$ complex Nd-L ${ }^{4}$ to yield the product as a yellow powder. Yield: $80 \%(0.090 \mathrm{~g})$. IR (KBrpellet, $\left.\mathrm{cm}^{-1}\right): 3446(\mathrm{~m}, \mathrm{v}(\mathrm{N}-\mathrm{H})), 2928,2854$ (s, aliphatic $\mathrm{C}-\mathrm{H}$ stretch), $2226(\mathrm{~m}, \mathrm{v}(\mathrm{C} \equiv \mathrm{N}))$. Calcd. for $\left(\mathrm{C}_{67} \mathrm{H}_{53} \mathrm{EuF}_{9} \mathrm{~N}_{5} \mathrm{O}_{8} \mathrm{~S}_{3}\right)\left(\mathrm{H}_{2} \mathrm{O}\right)$ : C 53.89, H 3.71, N 4.69. Found: C 53.55, H 3.68, N 4.78. ESI-MS (Acetonitrile, m/z): 1473.9, [M - H] $]^{-}$ 


\section{(I) Rhenium complex $\left[\operatorname{Re}(\mathrm{CO})_{3} \mathrm{BrL}_{\mathrm{II}}\right]$}

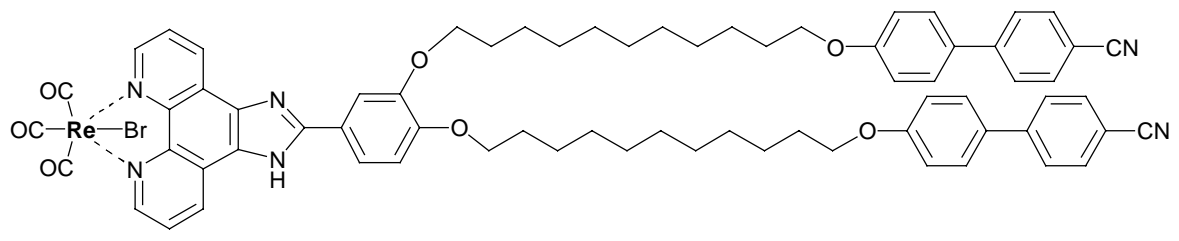

Rhenium(I) complex $\left[\operatorname{Re}(\mathrm{CO})_{3} \mathrm{Br} \mathbf{L}_{\text {II }}\right]$ was prepared by adding rhenium(I) pentacarbonyl bromide, $\operatorname{Re}(\mathrm{CO}){ }_{5} \mathrm{Br}(0.098 \mathrm{mmol}, 0.040 \mathrm{~g})$ to a solution of ligand $\mathbf{L}_{\mathbf{I I}}(0.098 \mathrm{mmol}, 0.100 \mathrm{~g})$ in toluene. The mixture was refluxed for 3 hours. Then the solvent was removed under reduced pressure and the crude product was purified on a silica column with $\mathrm{CHCl}_{3} / \mathrm{MeOH}$ (95:5) as the eluent. The complex was dissolved in a minimum amount of $\mathrm{CHCl}_{3}$ and precipitated in hexane. The yellow precipitate was filtered off and dried in a vacuum oven at $60{ }^{\circ} \mathrm{C}$. Yield: $75 \%\left(0.100\right.$ g). IR (KBr-pellet, $\left.\mathrm{cm}^{-1}\right)$ : $3430(\mathrm{~m}, \mathrm{v}(\mathrm{N}-\mathrm{H})), 2926,2853$ (s, aliphatic $\mathrm{C}-\mathrm{H}$ stretch), $2225(\mathrm{~m}, \mathrm{v}(\mathrm{C} \equiv \mathrm{N}))$. Calcd. for $\left(\mathrm{C}_{70} \mathrm{H}_{70} \mathrm{BrN}_{6} \mathrm{O}_{7} \mathrm{Re}\right)\left(\mathrm{H}_{2} \mathrm{O}\right): \mathrm{C} 60.42, \mathrm{H}$ 5.22, N 6.04. Found: C 60.52, H 5.18, N 6.00. ESI-MS (Acetonitrile, m/z): 1371.4, [M - H] 
(m) Yttrium complex of ligand [Y(tta $\left.)_{3} \mathrm{~L}_{\mathrm{II}}\right]$

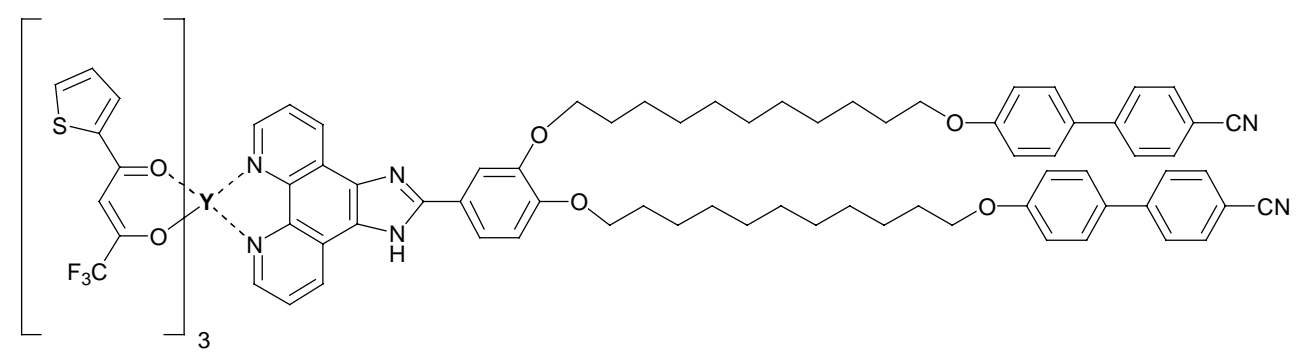

Yttrium(III) complex $\left[\mathrm{Y}(\mathrm{tta})_{3} \mathbf{L}_{\mathrm{II}}\right]$ was prepared by adding a solution of $\mathrm{Y}(\mathrm{tta})_{3} \cdot 2 \mathrm{H}_{2} \mathrm{O}(0.098$ mmol, 0.077g) in $\mathrm{CHCl}_{3}$ to a solution of ligand $\mathbf{L}_{\mathbf{I I}}(0.098 \mathrm{mmol}, 0.100 \mathrm{~g})$ in $\mathrm{CHCl}_{3}$. The mixture was refluxed for 1 hour. Then the solvent was removed under reduced pressure. The product was dissolved in a minimum amount of $\mathrm{CHCl}_{3}$ and precipitated in hexane. The yellow precipitate was filtered off and dried in a vacuum oven at $60{ }^{\circ} \mathrm{C}$. Yield: $88 \%(0.154$

g). IR (KBr-pellet, $\left.\mathrm{cm}^{-1}\right)$ : $3406(\mathrm{~m}, \mathrm{v}(\mathrm{N}-\mathrm{H})), 2927,2854$ (s, aliphatic C-H stretch), 2225 (m, $v(\mathrm{C} \equiv \mathrm{N})$ ). Calcd. for $\left(\mathrm{C}_{91} \mathrm{H}_{82} \mathrm{~F}_{9} \mathrm{~N}_{6} \mathrm{O}_{10} \mathrm{~S}_{3} \mathrm{Y}\right)\left(\mathrm{H}_{2} \mathrm{O}\right)$ : $\mathrm{C}$ 60.93, $\mathrm{H} 4.72, \mathrm{~N}$ 4.69. Found: $\mathrm{C}$ 61.22, H 4.70, N 4.62. ESI-MS (Acetonitrile, m/z): 1774.1, [M - H] ${ }^{-}$. 


\section{(n) Lanthanum complex $\left[\mathrm{La}(\mathrm{tta})_{3} \mathrm{~L}_{\mathrm{II}}\right]$}

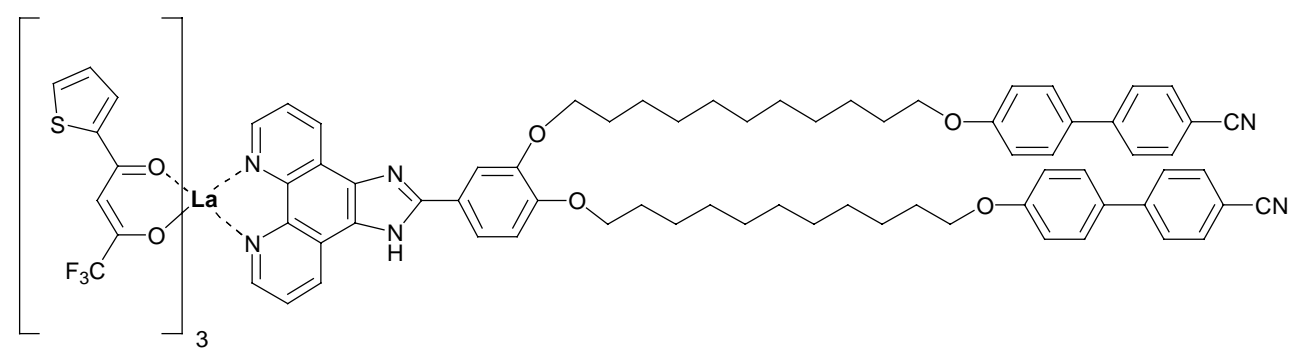

Lanthanum(III) complex $\left[\mathrm{La}(\mathrm{tta})_{3} \mathbf{L}_{\mathbf{I I}}\right]$ was prepared starting from ligand $\mathbf{L}_{\mathbf{I I}}(0.098 \mathrm{mmol}$, $0.100 \mathrm{~g})$ and $\mathrm{La}(\mathrm{tta})_{3} \cdot 2 \mathrm{H}_{2} \mathrm{O}(0.098 \mathrm{mmol}, 0.082 \mathrm{~g})$ using the procedure described for the $\mathrm{Y}$ complex to yield the product as a yellow powder. Yield: $79 \%(0.141 \mathrm{~g})$. IR (KBr-pellet, $\mathrm{cm}^{-}$ $\left.{ }^{1}\right): 3411(\mathrm{~m}, \mathrm{v}(\mathrm{N}-\mathrm{H})), 2927,2854$ (s, aliphatic $\mathrm{C}-\mathrm{H}$ stretch), $2225(\mathrm{~m}, \mathrm{v}(\mathrm{C} \equiv \mathrm{N}))$. Calcd. for $\left(\mathrm{C}_{91} \mathrm{H}_{82} \mathrm{~F}_{9} \mathrm{LaN}_{6} \mathrm{O}_{10} \mathrm{~S}_{3}\right)\left(\mathrm{H}_{2} \mathrm{O}\right): \mathrm{C} 59.28, \mathrm{H} 4.59, \mathrm{~N}$ 4.56. Found: C 58.85, H 4.49, N 4.26. ESIMS (Acetonitrile, m/z): 1824.2, [M - H] $]^{-}$ 


\section{(o) Neodymium complex $\left[\mathrm{Nd}(\mathrm{tta})_{3} \mathrm{~L}_{\mathrm{II}}\right]$}

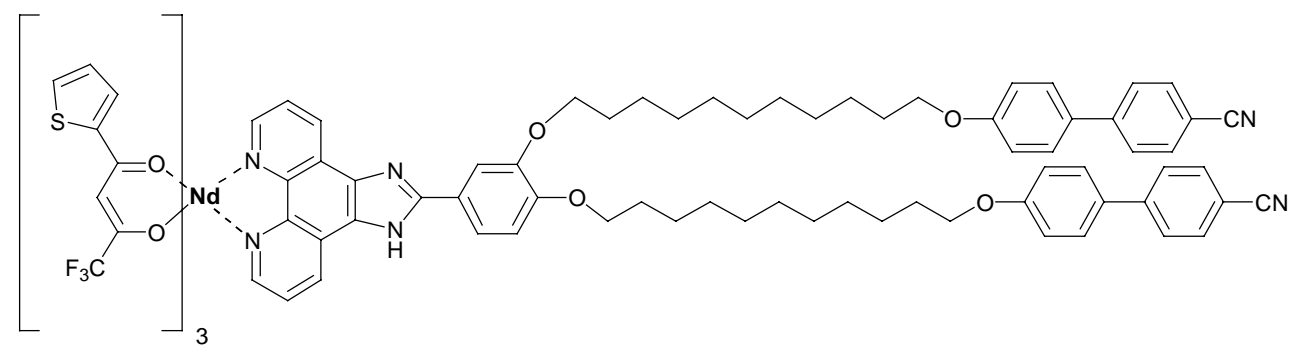

Neodymium(III) complex $\left[\mathrm{Nd}(\mathrm{tta})_{3} \mathbf{L}_{\mathbf{I I}}\right]$ was prepared starting from ligand $\mathbf{L}_{\mathbf{I I}}(0.098 \mathrm{mmol}$, $0.100 \mathrm{~g})$ and $\mathrm{Nd}(\mathrm{tta})_{3} \cdot 2 \mathrm{H}_{2} \mathrm{O}(0.098 \mathrm{mmol}, 0.082 \mathrm{~g})$ using the procedure described for the $\mathrm{Y}$ complex to yield the product as a yellow powder. Yield: $84 \%(0.150 \mathrm{~g})$. IR (KBr-pellet, $\mathrm{cm}^{-}$ $\left.{ }^{1}\right): 3431(\mathrm{~m}, \mathrm{v}(\mathrm{N}-\mathrm{H})), 2927,2854$ (s, aliphatic $\mathrm{C}-\mathrm{H}$ stretch), $2228(\mathrm{~m}, \mathrm{v}(\mathrm{C} \equiv \mathrm{N}))$. Calcd. for $\left(\mathrm{C}_{91} \mathrm{H}_{82} \mathrm{~F}_{9} \mathrm{~N}_{6} \mathrm{NdO}_{10} \mathrm{~S}_{3}\right)\left(\mathrm{H}_{2} \mathrm{O}\right)$ : C 59.11, H 4.58, N 4.54. Found: C 59.16, H 4.61, N 4.43. ESIMS (Acetonitrile, m/z): 1829.9, [M - H] $]^{-}$

\section{(p) Samarium complex $\left[\mathrm{Sm}(\mathrm{tta})_{3} \mathbf{L}_{\mathrm{II}}\right]$}

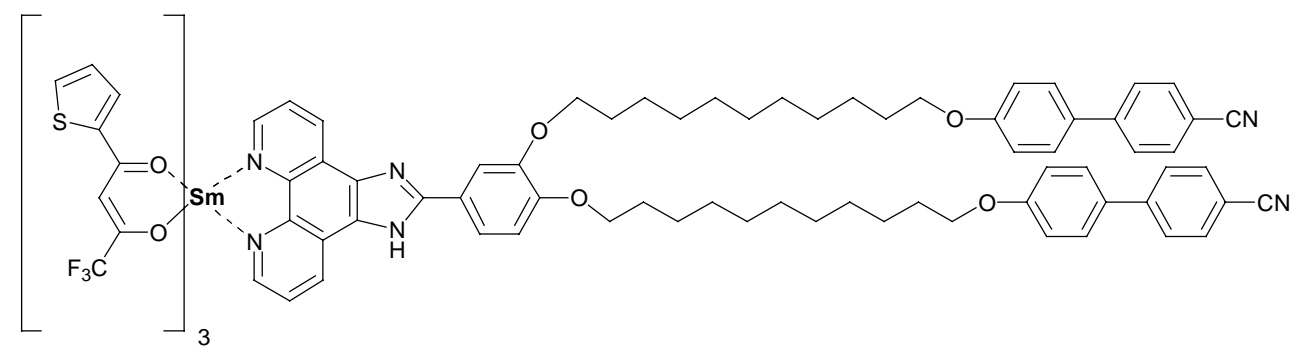

Samarium(III) complex [Sm(tta) $\left.)_{3} \mathbf{L}_{\mathbf{I I}}\right]$ was prepared starting from ligand $\mathbf{L}_{\mathbf{I I}}(0.098 \mathrm{mmol}$, $0.100 \mathrm{~g})$ and $\mathrm{Sm}(\mathrm{tta})_{3} \cdot 2 \mathrm{H}_{2} \mathrm{O}(0.098 \mathrm{mmol}, 0.083 \mathrm{~g})$ using the procedure described for the $\mathrm{Y}$ complex to yield the product as a yellow powder. Yield: $81 \%(0.146 \mathrm{~g})$. IR (KBr-pellet, $\mathrm{cm}^{-}$ $\left.{ }^{1}\right): 3421(\mathrm{~m}, v(\mathrm{~N}-\mathrm{H})), 2927,2854$ (s, aliphatic $\mathrm{C}-\mathrm{H}$ stretch), $2225(\mathrm{~m}, \mathrm{v}(\mathrm{C} \equiv \mathrm{N}))$. Calcd. for $\left(\mathrm{C}_{91} \mathrm{H}_{82} \mathrm{~F}_{9} \mathrm{~N}_{6} \mathrm{O}_{10} \mathrm{~S}_{3} \mathrm{Sm}\right)\left(\mathrm{H}_{2} \mathrm{O}\right): \mathrm{C} 58.91, \mathrm{H} 4.56, \mathrm{~N}$ 4.53. Found: C 59.13, H 4.47, N 4.40. ESIMS (Acetonitrile, m/z): 1835.7, [M - H] $]^{-}$ 


\section{(q) Europium complex $\left[\mathrm{Eu}(\mathrm{tta})_{3} \mathrm{~L}_{\mathrm{II}}\right]$}

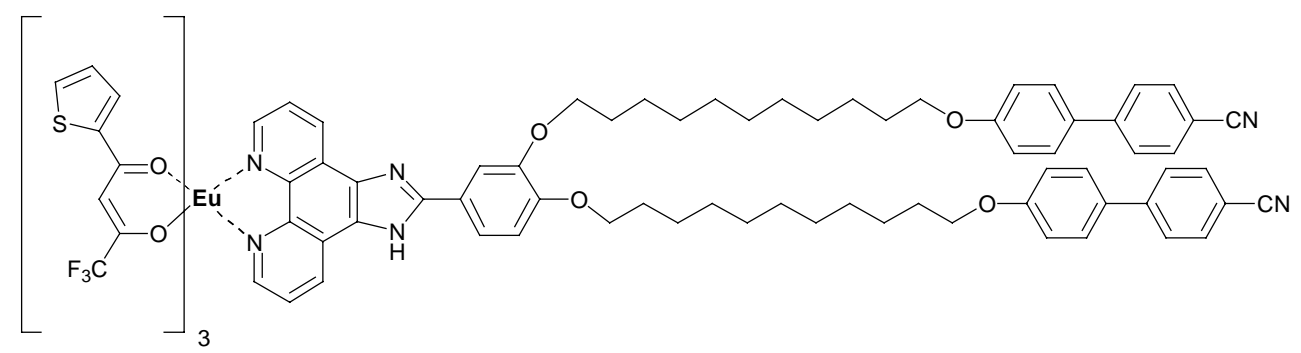

Europium(III) complex $\left[\mathrm{Eu}(\mathrm{tta})_{3} \mathbf{L}_{\mathbf{I I}}\right]$ was prepared starting from ligand $\mathbf{L}_{\mathbf{I I}}(0.098 \mathrm{mmol}$,

$0.100 \mathrm{~g})$ and $\mathrm{Eu}(\mathrm{tta})_{3} \cdot 2 \mathrm{H}_{2} \mathrm{O}(0.098 \mathrm{mmol}, 0.084 \mathrm{~g})$ using the procedure described for the $\mathrm{Y}$ complex to yield the product as a yellow powder. Yield: $85 \%(0.150 \mathrm{~g})$. IR (KBr-pellet, $\mathrm{cm}^{-}$ $\left.{ }^{1}\right): 3431(\mathrm{~m}, \mathrm{v}(\mathrm{N}-\mathrm{H})), 2926,2853$ (s, aliphatic $\mathrm{C}-\mathrm{H}$ stretch), $2225(\mathrm{~m}, \mathrm{v}(\mathrm{C} \equiv \mathrm{N}))$. Calcd. for $\left(\mathrm{C}_{91} \mathrm{H}_{82} \mathrm{EuF}_{9} \mathrm{~N}_{6} \mathrm{O}_{10} \mathrm{~S}_{3}\right)\left(\mathrm{H}_{2} \mathrm{O}\right): \mathrm{C} 58.86, \mathrm{H} 4.56, \mathrm{~N}$ 4.53. Found: $\mathrm{C}$ 59.30, H 4.08, N 4.38. ESIMS (Acetonitrile, m/z): 1836.9, [M - H] . 


\section{(r) Erbium complex $\left[\operatorname{Er}(\operatorname{tta})_{3} \mathrm{~L}_{\mathrm{II}}\right]$}

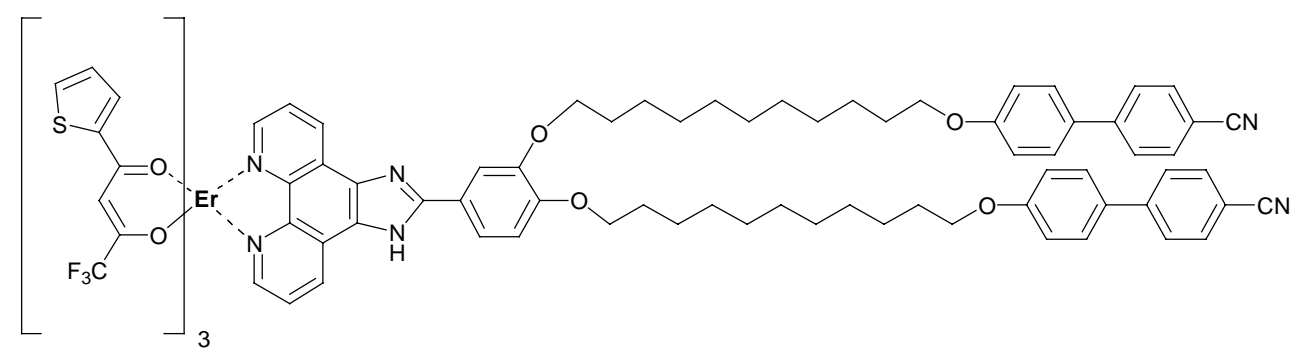

Erbium(III) complex[Er(tta) $\left.\mathbf{L}_{\mathbf{I I}}\right]$ was prepared starting from ligand $\mathbf{L}_{\mathbf{I I}}(0.098 \mathrm{mmol}, 0.100$ g) and $\mathrm{Er}(\mathrm{tta})_{3} \cdot 2 \mathrm{H}_{2} \mathrm{O}(0.098 \mathrm{mmol}, 0.085 \mathrm{~g})$ using the procedure described for the $\mathrm{Y}$ complex to yield the product as a yellow powder. Yield: $81 \%(0.147 \mathrm{~g})$. IR (KBr-pellet, $\mathrm{cm}^{-}$ $\left.{ }^{1}\right): 3406(\mathrm{~m}, \mathrm{v}(\mathrm{N}-\mathrm{H})), 2927,2854$ (s, aliphatic $\mathrm{C}-\mathrm{H}$ stretch), $2225(\mathrm{~m}, \mathrm{v}(\mathrm{C} \equiv \mathrm{N}))$. Calcd. for $\left(\mathrm{C}_{91} \mathrm{H}_{82} \mathrm{ErF}_{9} \mathrm{~N}_{6} \mathrm{O}_{10} \mathrm{~S}_{3}\right)\left(\mathrm{H}_{2} \mathrm{O}\right): \mathrm{C}$ 58.38, $\mathrm{H}$ 4.52, N 4.49. Found: C 58.73, H 4.47, N 4.43. ESIMS (Acetonitrile, m/z): 1851.1, [M - H] $]^{-}$ 


\section{(s) Ytterbium complex $\left[\mathrm{Yb}(\mathrm{tta})_{3} \mathrm{~L}_{\mathrm{II}}\right]$}

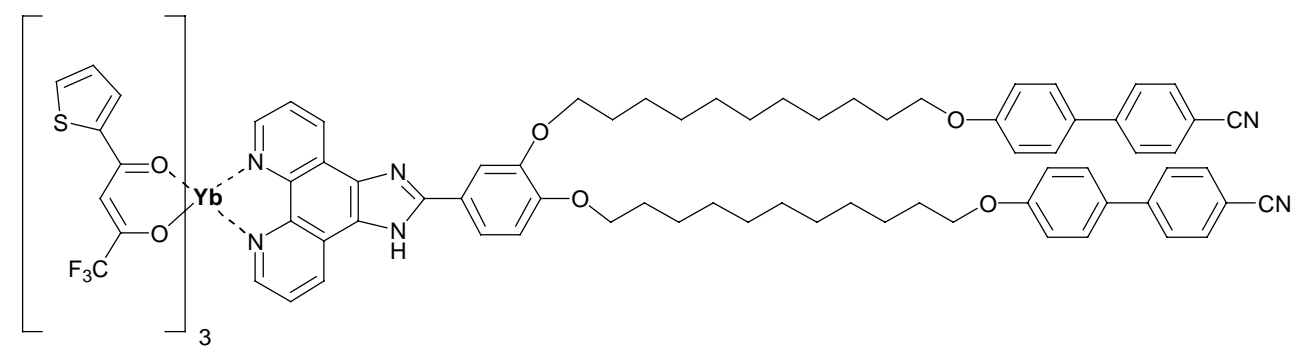

Ytterbium(III) complex [Yb(tta) $\left.{ }_{3} \mathbf{L}_{\mathbf{I I}}\right]$ was prepared starting from ligand $\mathbf{L}_{\mathbf{I I}}(0.098 \mathrm{mmol}$, $0.100 \mathrm{~g})$ and $\mathrm{Yb}(\mathrm{tta})_{3} \cdot 2 \mathrm{H}_{2} \mathrm{O}(0.098 \mathrm{mmol}, 0.086 \mathrm{~g})$ using the procedure described for the $\mathrm{Y}$ complex to yield the product as a yellow powder. Yield: $84 \%(0.153 \mathrm{~g})$. IR (KBr-pellet, $\mathrm{cm}^{-}$ $\left.{ }^{1}\right): 3421(\mathrm{~m}, \mathrm{v}(\mathrm{N}-\mathrm{H})), 2927,2854$ (s, aliphatic $\mathrm{C}-\mathrm{H}$ stretch), $2225(\mathrm{~m}, \mathrm{v}(\mathrm{C} \equiv \mathrm{N}))$. Calcd. for $\left(\mathrm{C}_{91} \mathrm{H}_{82} \mathrm{~F}_{9} \mathrm{~N}_{6} \mathrm{O}_{10} \mathrm{~S}_{3} \mathrm{Yb}\right)\left(\mathrm{H}_{2} \mathrm{O}\right): \mathrm{C}$ 58.20, H 4.51, N 4.48. Found: C 58.28, H 4.42, N 4.29. ESIMS (Acetonitrile, m/z): 1857.8, [M - H] $]^{-}$ 


\section{(t) Rhenium complex $\left[\operatorname{Re}(\mathrm{CO})_{3} \mathrm{BrL}_{\mathrm{III}}\right]$}

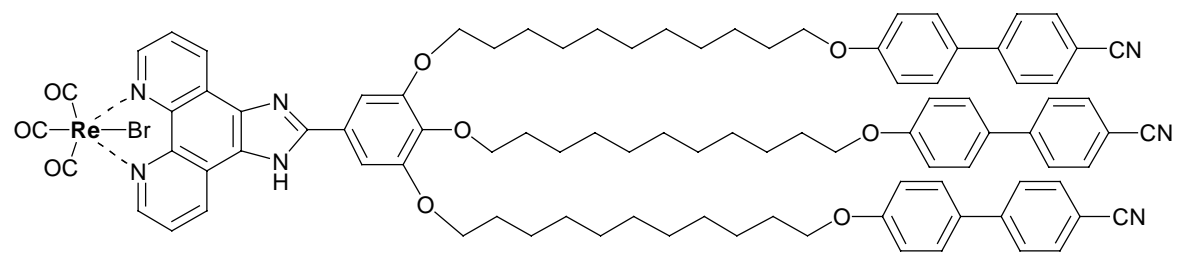

Rhenium(I) complex $\left[\operatorname{Re}(\mathrm{CO})_{3} \mathrm{BrL}_{\mathrm{III}}\right]$ was prepared by adding rhenium pentacarbonyl bromide, $\operatorname{Re}(\mathrm{CO})_{5} \mathrm{Br}(0.043 \mathrm{mmol}, 0.018 \mathrm{~g})$ to a solution of ligand $\mathbf{L}_{\mathrm{III}}(0.043 \mathrm{mmol}, 0.060$ $\mathrm{g}$ ) in toluene. The mixture was refluxed for 3 hours. Then the solvent was removed under reduced pressure and the crude product was purified on a silica column with $\mathrm{CHCl}_{3} / \mathrm{MeOH}$ (95:5) as the eluent. The complex was dissolved in a minimum amount of $\mathrm{CHCl}_{3}$ and precipitated in hexane. The yellow precipitate was filtered off and dried in a vacuum oven at $60{ }^{\circ} \mathrm{C}$. Yield: $47 \%$ (0.035 g). IR (KBr-pellet, $\left.\mathrm{cm}^{-1}\right): 3446(\mathrm{~m}, \mathrm{v}(\mathrm{N}-\mathrm{H})), 2922,2850$ (s, aliphatic $\mathrm{C}-\mathrm{H}$ stretch), $2224(\mathrm{~m}, \mathrm{v}(\mathrm{C} \equiv \mathrm{N}))$. Calcd. for $\left(\mathrm{C}_{94} \mathrm{H}_{99} \mathrm{BrN}_{7} \mathrm{O}_{9} \mathrm{Re}\right)\left(\mathrm{H}_{2} \mathrm{O}\right): \mathrm{C} 64.33, \mathrm{H}$ 5.80, N 5.59. Found: C 64.12, H 5.69, N 5.42. ESI-MS (Acetonitrile, m/z): 1734.4, [M - H] $]^{-}$ 


\section{(u) Europium complex $\left[\mathrm{Eu}(\mathrm{tta})_{3} \mathrm{~L}_{\mathrm{III}}\right]$}

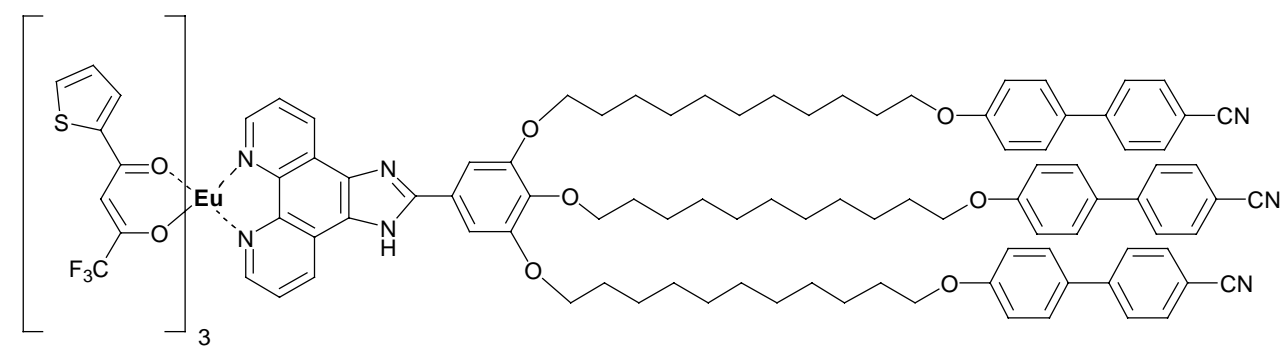

Europium(III) complex [Eu(tta) $)_{3} \mathbf{L}_{\text {III }}$ was prepared by adding a solution of $\mathrm{Eu}(\mathrm{tta})_{3} \cdot 2 \mathrm{H}_{2} \mathrm{O}$ (0.054 mmol, $0.046 \mathrm{~g})$ in $\mathrm{CHCl}_{3}$ to a solution of ligand $\mathbf{L}_{\mathrm{III}}(0.054 \mathrm{mmol}, 0.075 \mathrm{~g})$ in $\mathrm{CHCl}_{3}$. The mixture was refluxed for 1 hour. Then the solvent was removed under reduced pressure. The product was dissolved in a minimum amount of $\mathrm{CHCl}_{3}$ and precipitated in hexane. The yellow precipitate was filtered off and dried in a vacuum oven at $60{ }^{\circ} \mathrm{C}$. Yield: $88 \%(0.105$ g). IR (KBr-pellet, $\left.\mathrm{cm}^{-1}\right): 3448(\mathrm{~m}, \mathrm{v}(\mathrm{N}-\mathrm{H})), 2926,2852$ (s, aliphatic C-H stretch), 2226 (m, $v(\mathrm{C} \equiv \mathrm{N}))$. Calcd. for $\left(\mathrm{C}_{115} \mathrm{H}_{111} \mathrm{EuF}_{9} \mathrm{~N}_{7} \mathrm{O}_{12} \mathrm{~S}_{3}\right)\left(\mathrm{H}_{2} \mathrm{O}\right): \mathrm{C}$ 62.21, H 5.13, $\mathrm{N}$ 4.42. Found: $\mathrm{C}$ 62.41, H 5.18, N 4.58. ESI-MS (Acetonitrile, m/z): 1099.7, [M - 2H $]^{2-}$. 


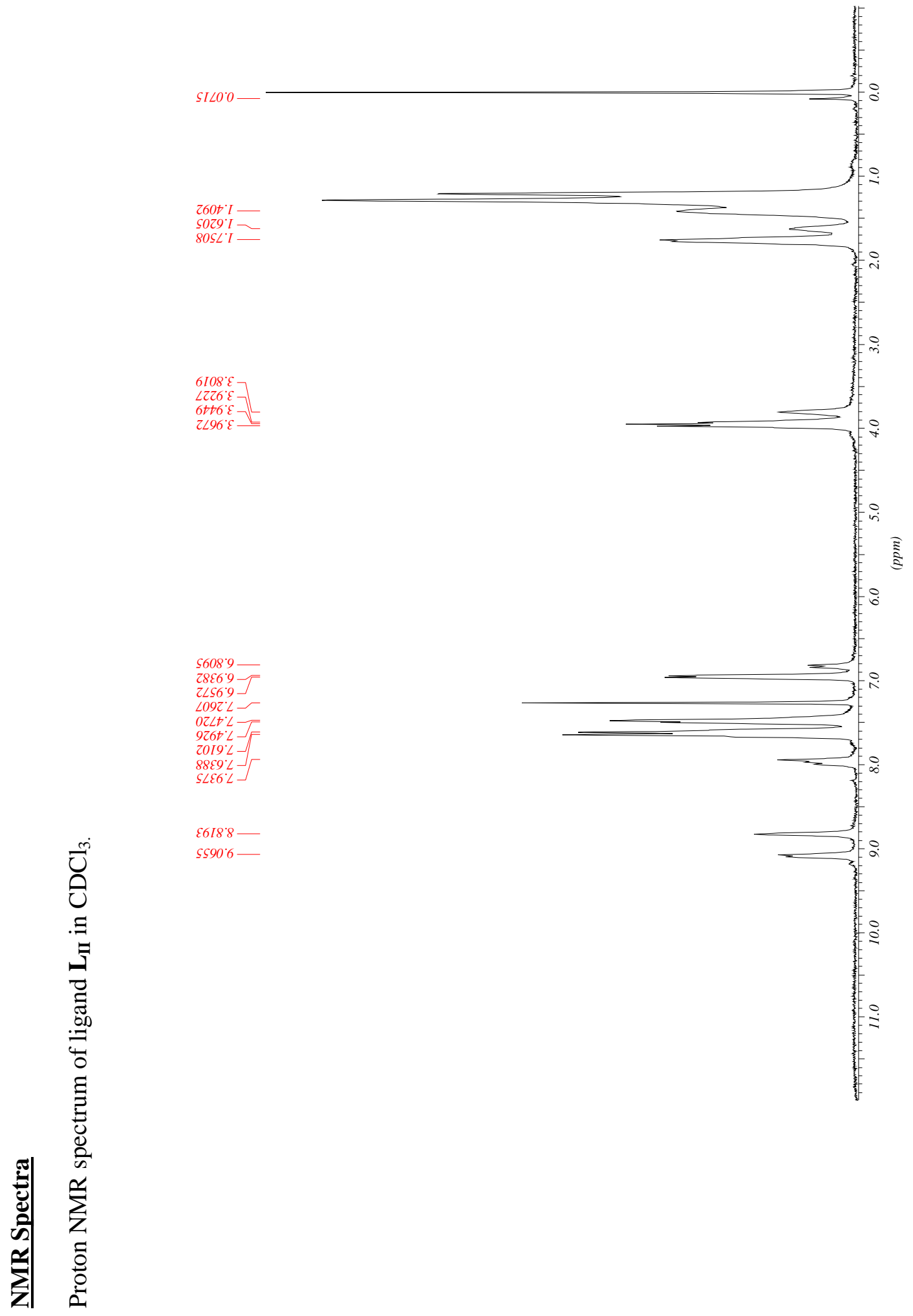




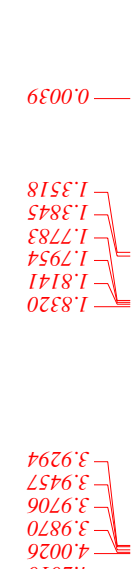

OIOZ't
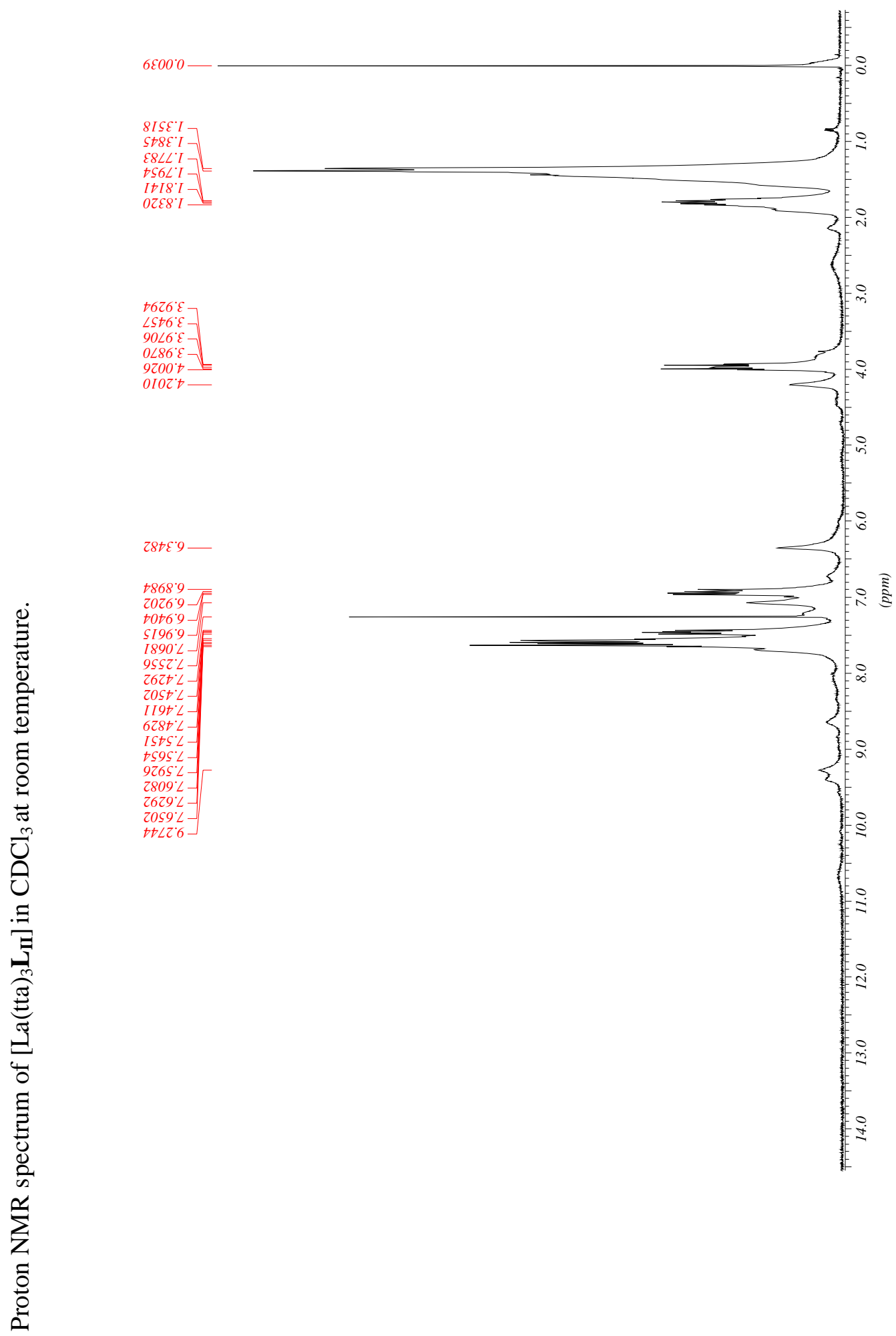


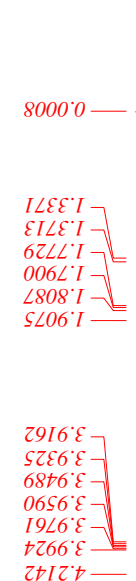

$\angle S E E^{\circ} 9$

$9986^{\circ}$
$\varepsilon 8566^{\circ}$

$\begin{aligned} & \mathcal{E} 856^{\circ} 9- \\ & \varepsilon \angle 90^{\circ} \mathrm{L}\end{aligned}-$

$E \angle 90^{\circ} \mathrm{L}$
$S 6 S Z^{\circ} \mathrm{L}$

zzzt' $L$

Ott ${ }^{\circ} \mathrm{L}$

$9290^{\circ} \mathrm{L}-$

I8ES $\mathrm{L}$

I6SS' $\mathrm{L}$

$6+6 S S^{\circ} \angle$

$7800^{\circ}$

C8 $80^{\circ}$

$8099^{\circ} \mathrm{L}$

$\mathcal{E} 099^{\circ} \mathrm{L}$
$\varepsilon+\mathcal{E} \mathcal{C}^{\circ} 6$

气ั

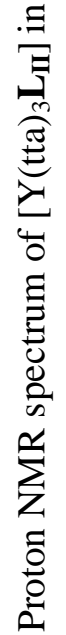




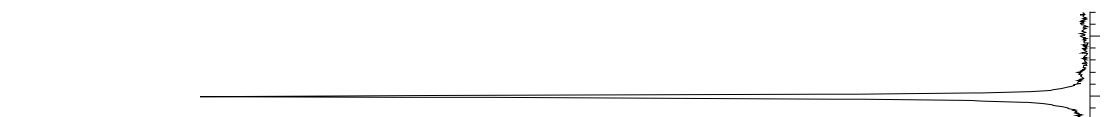

$9666^{\circ} \mathcal{E}$

$916 I^{\circ} t$

oeEt

苟

$2296^{\circ} 9$

$8 \angle 869-5$

$8290^{\circ} \mathrm{L}$

$806 t^{\circ} \mathrm{L}-$

$S+I S^{\circ} \mathrm{L}-$
$I 929^{\circ} \mathrm{L}$

ESS9 $\mathrm{L}$

I $288^{\circ} \mathrm{L}$

LIt 18

21998

02568

$2220 \%$

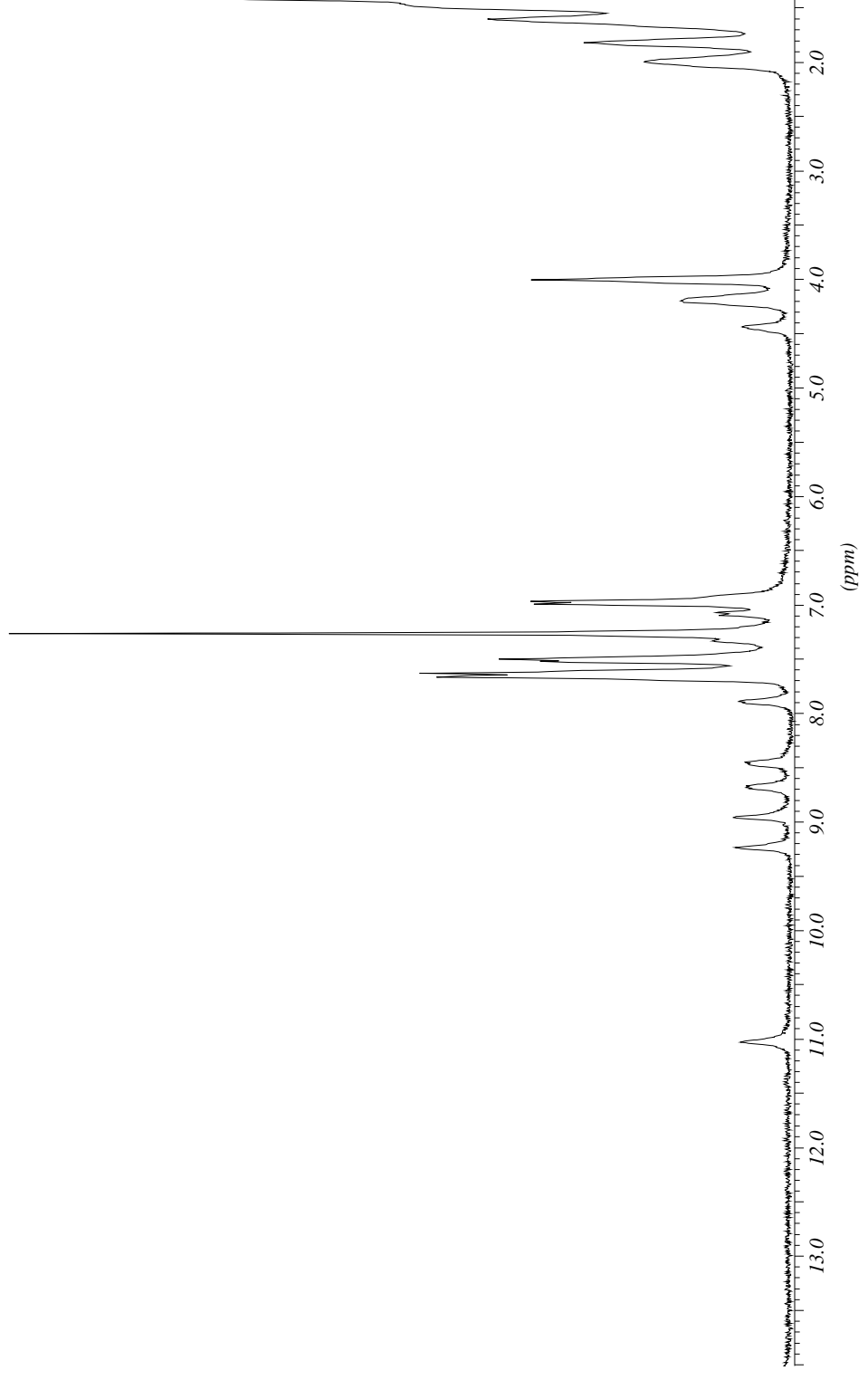




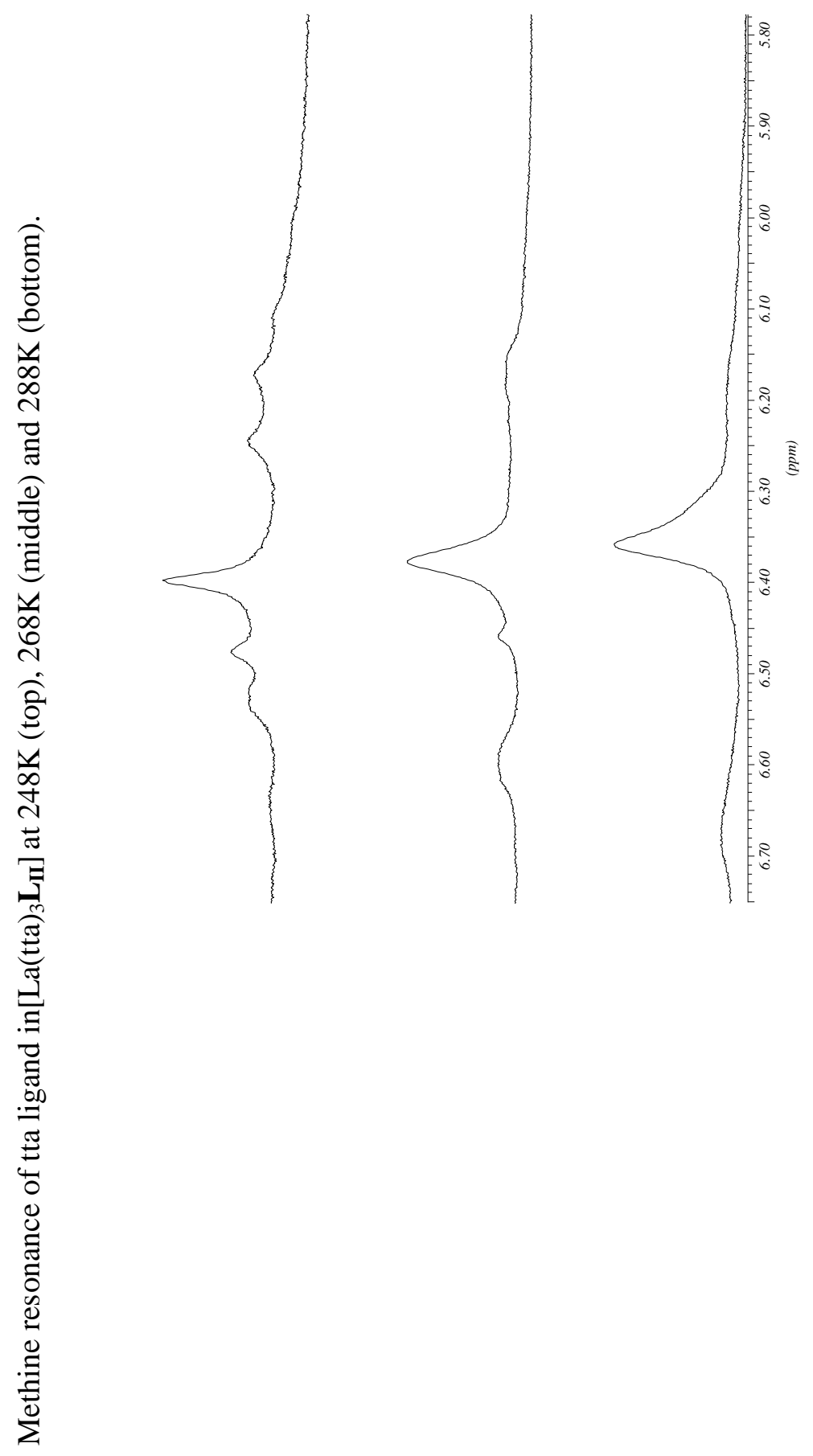



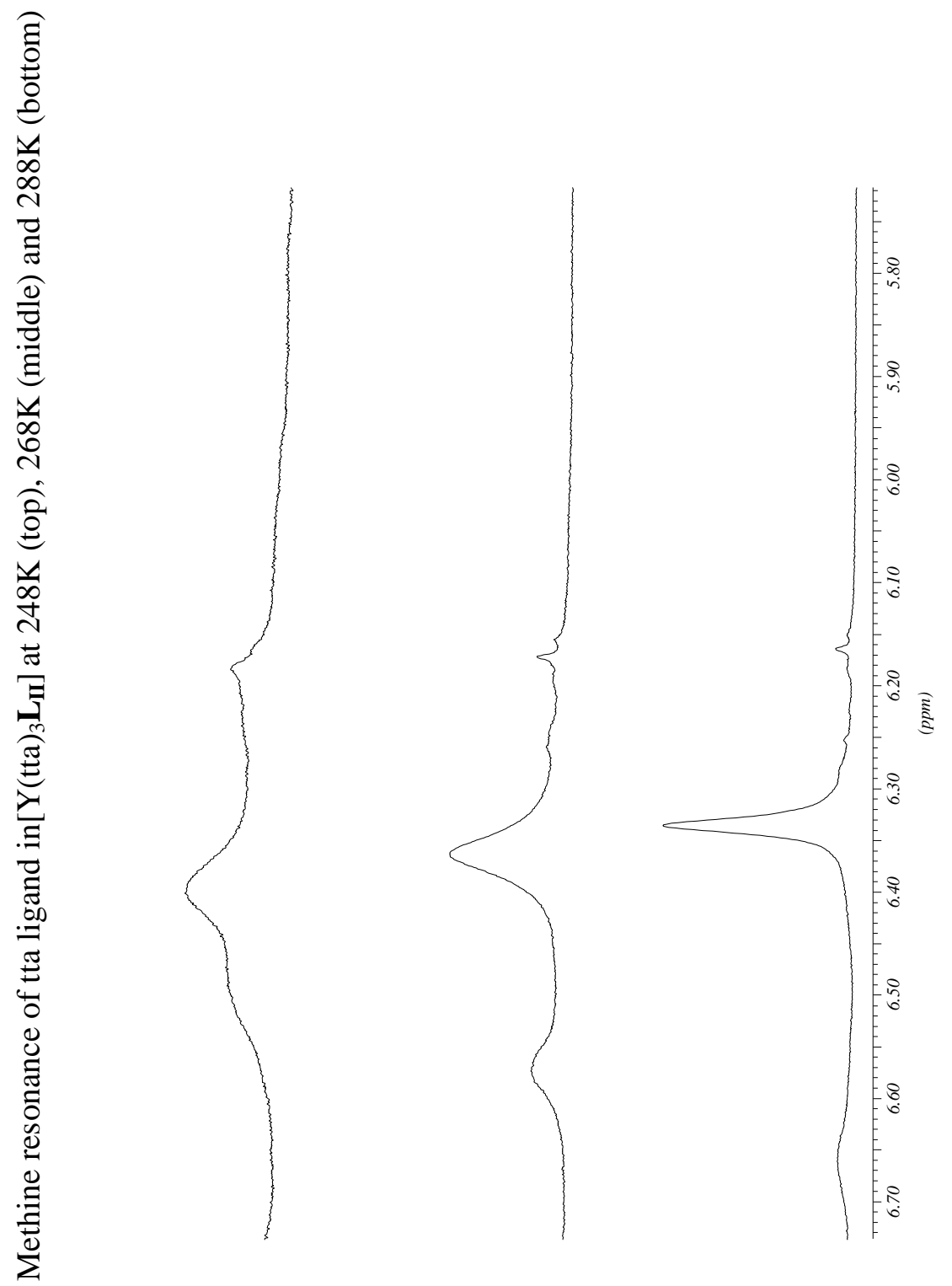


\section{Microscope textures}
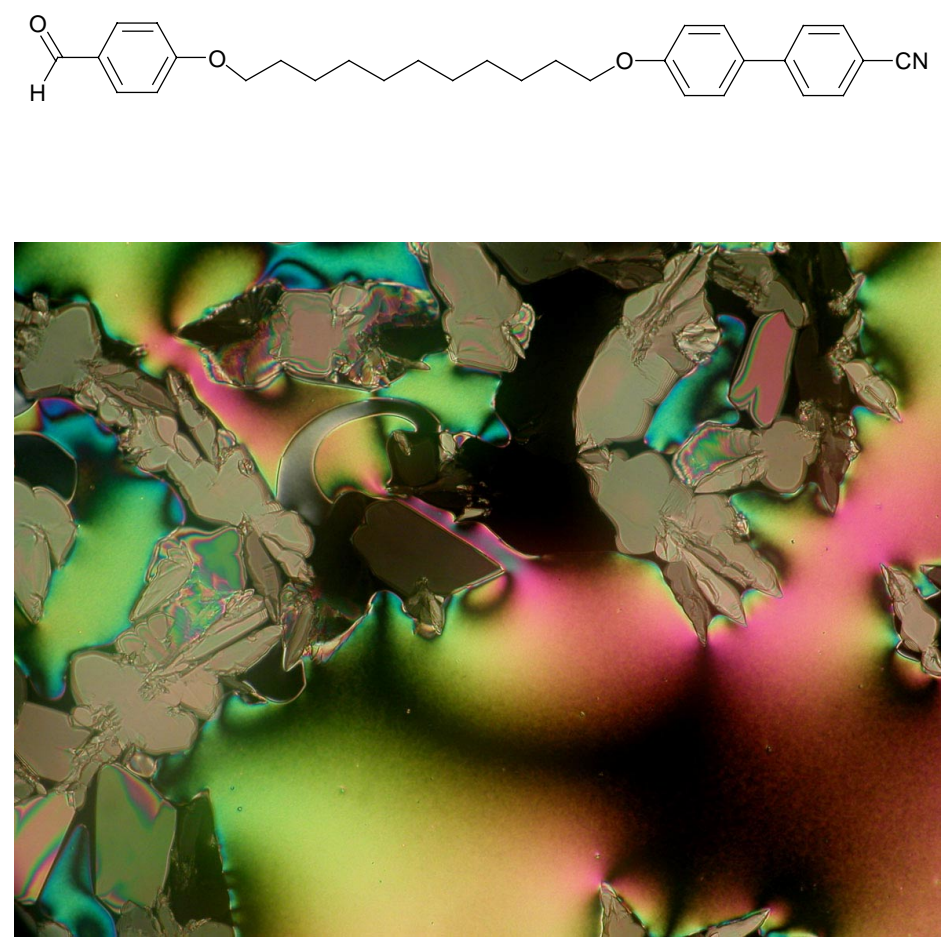

Optical texture of the nematic phase upon cooling at $85^{\circ} \mathrm{C}$. The compound has partially crystallized out. 

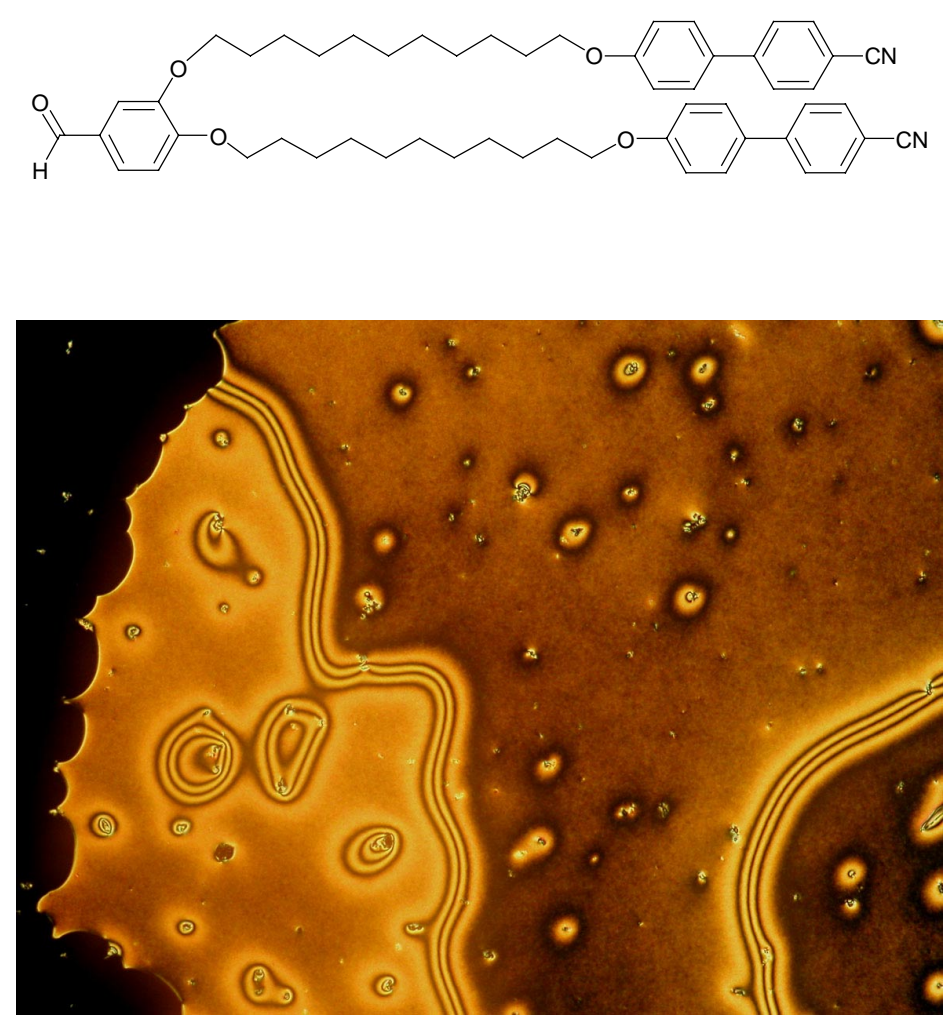

Optical texture of the nematic phase upon cooling at $80{ }^{\circ} \mathrm{C}$. 

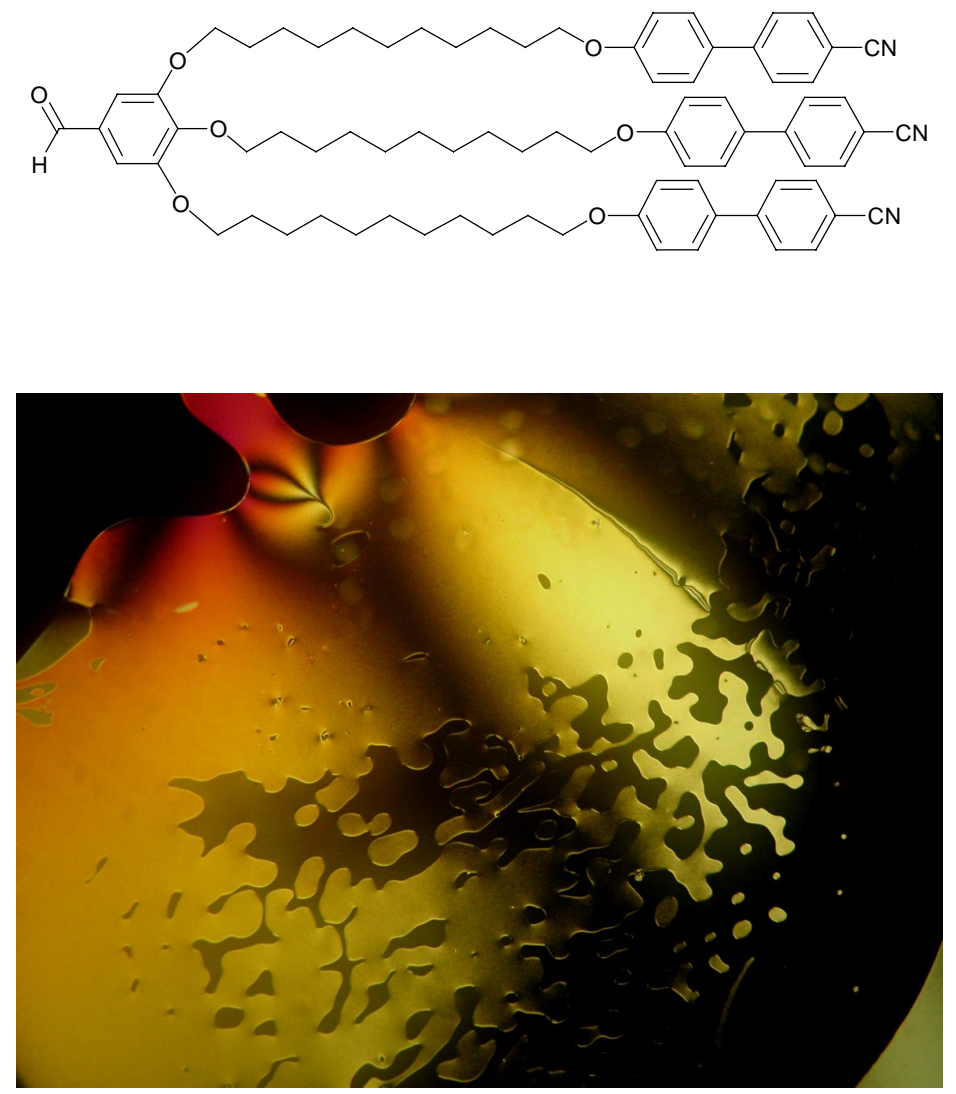

Optical texture of the nematic phase upon cooling at $110{ }^{\circ} \mathrm{C}$. 

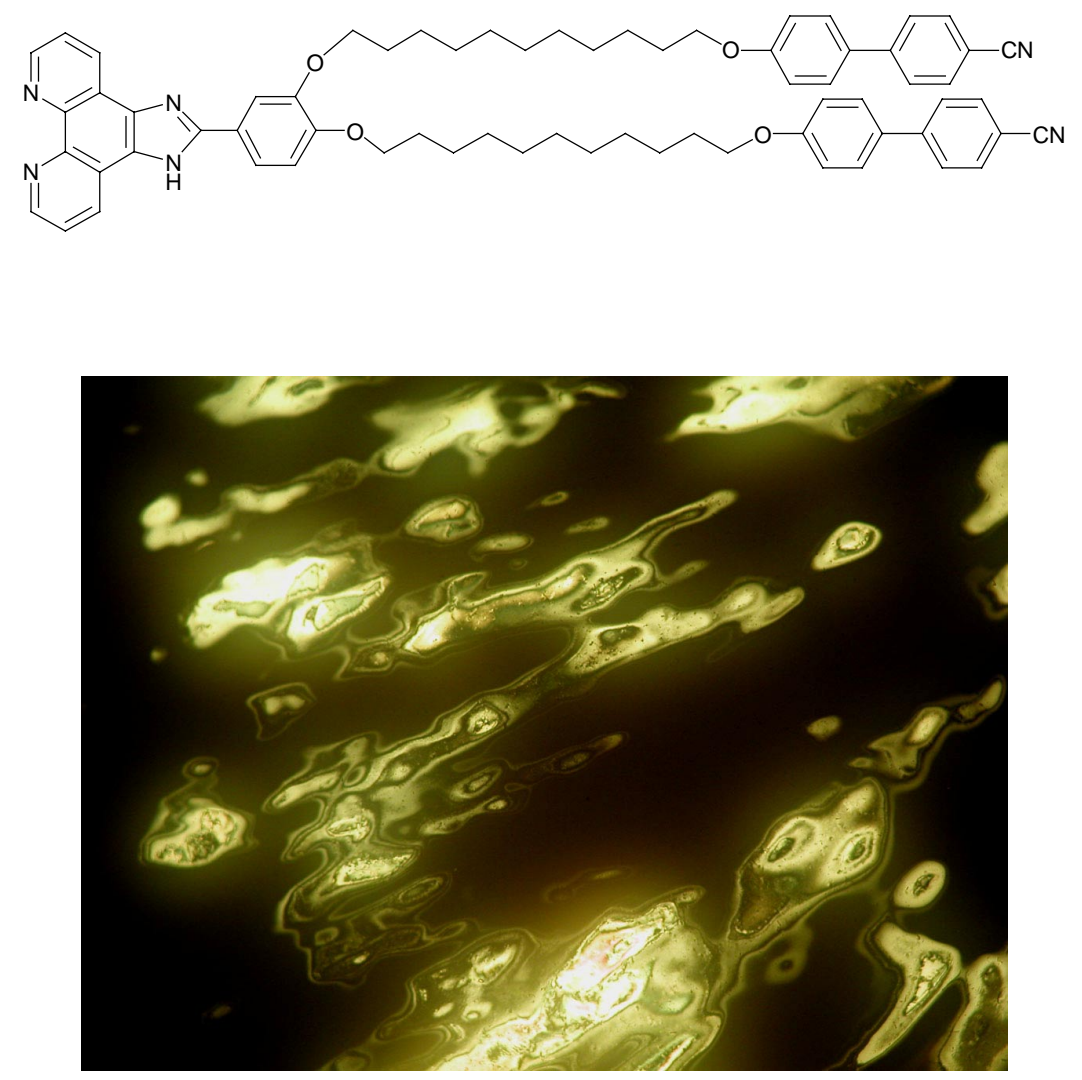

Optical texture of the nematic phase upon cooling at $105^{\circ} \mathrm{C}$. Large homeotropic areas are visible. 

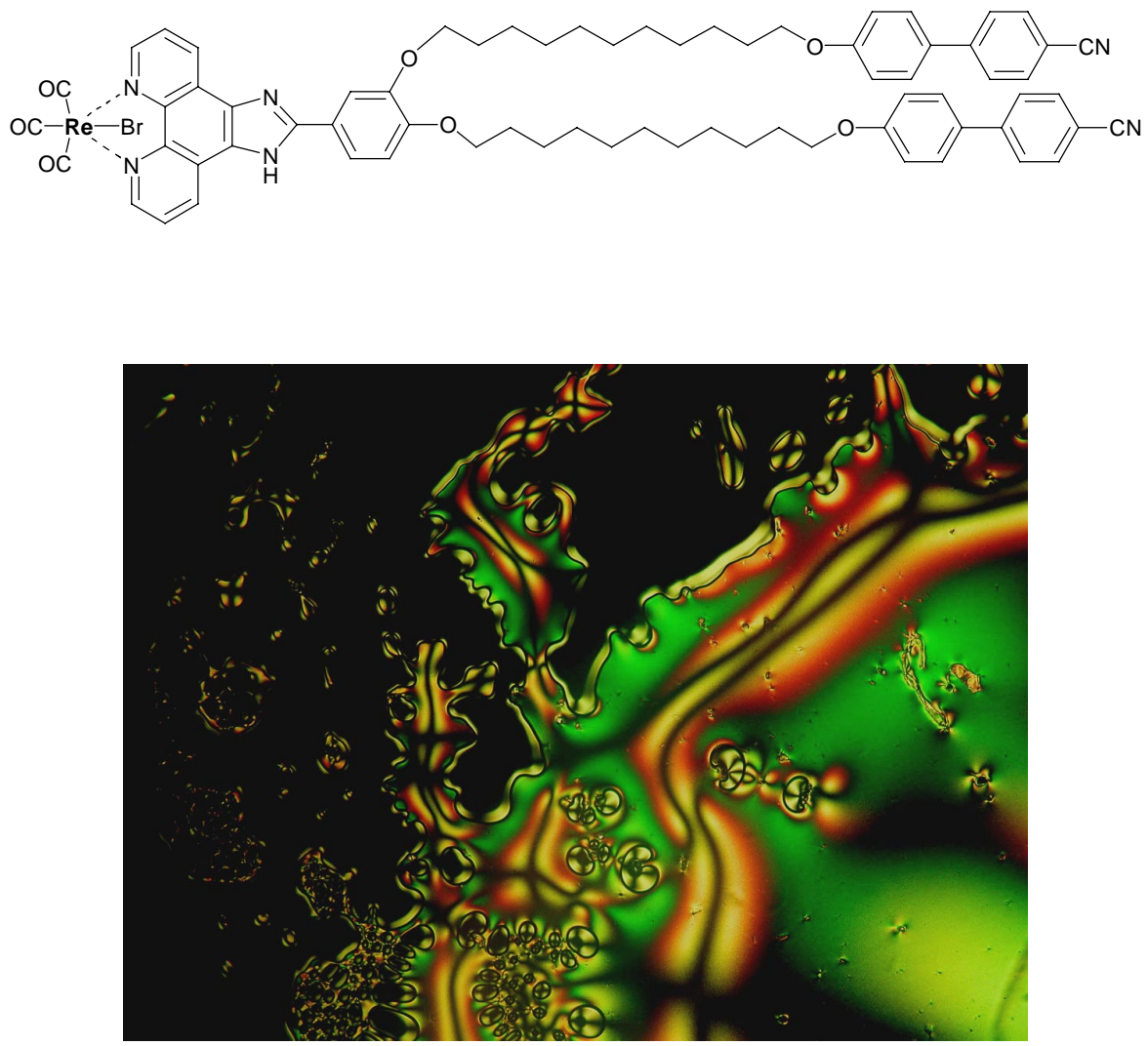

Optical texture of the nematic phase upon cooling at $100{ }^{\circ} \mathrm{C}$. 

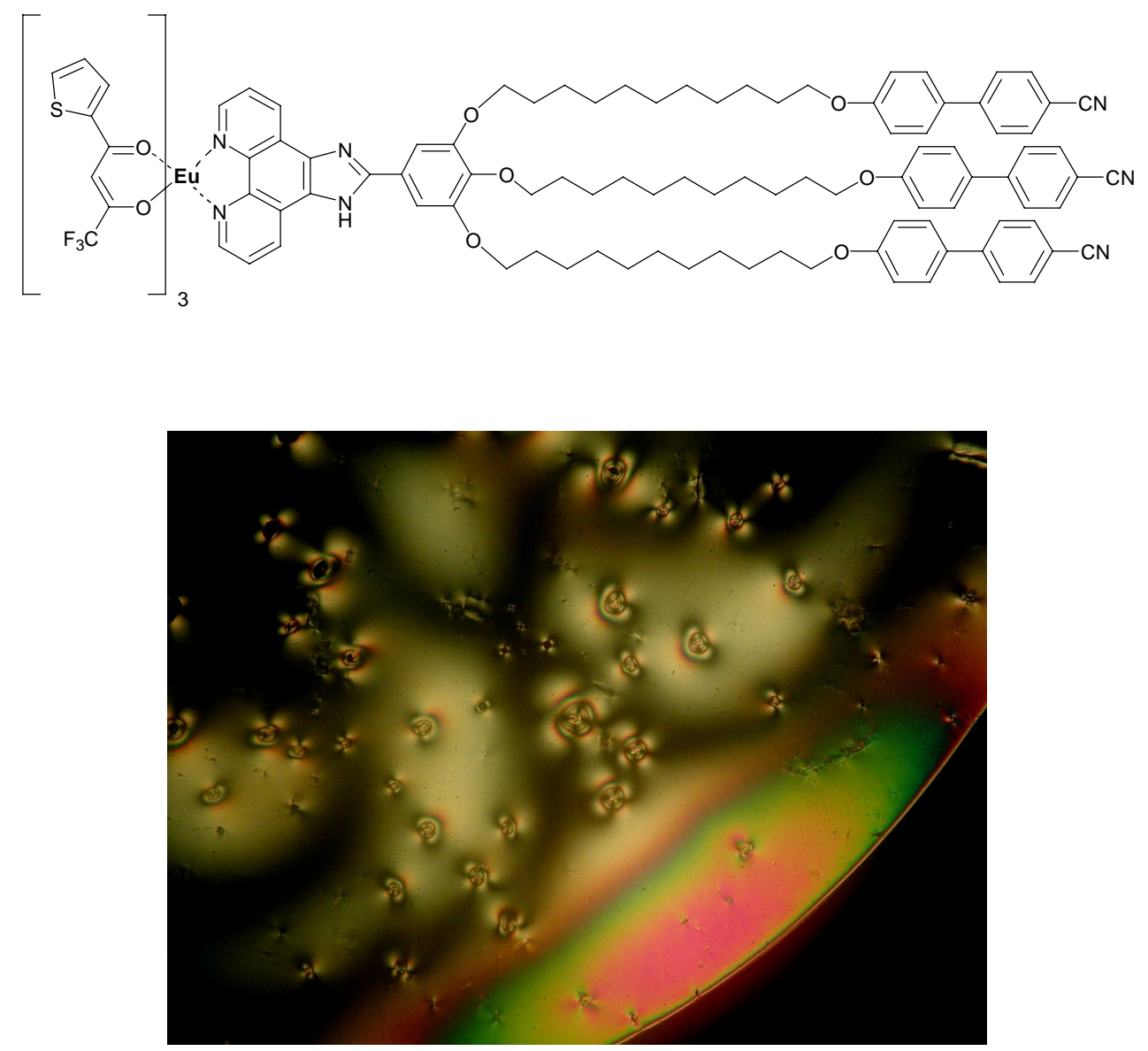

Optical texture of the nematic phase upon cooling at $85{ }^{\circ} \mathrm{C}$. 

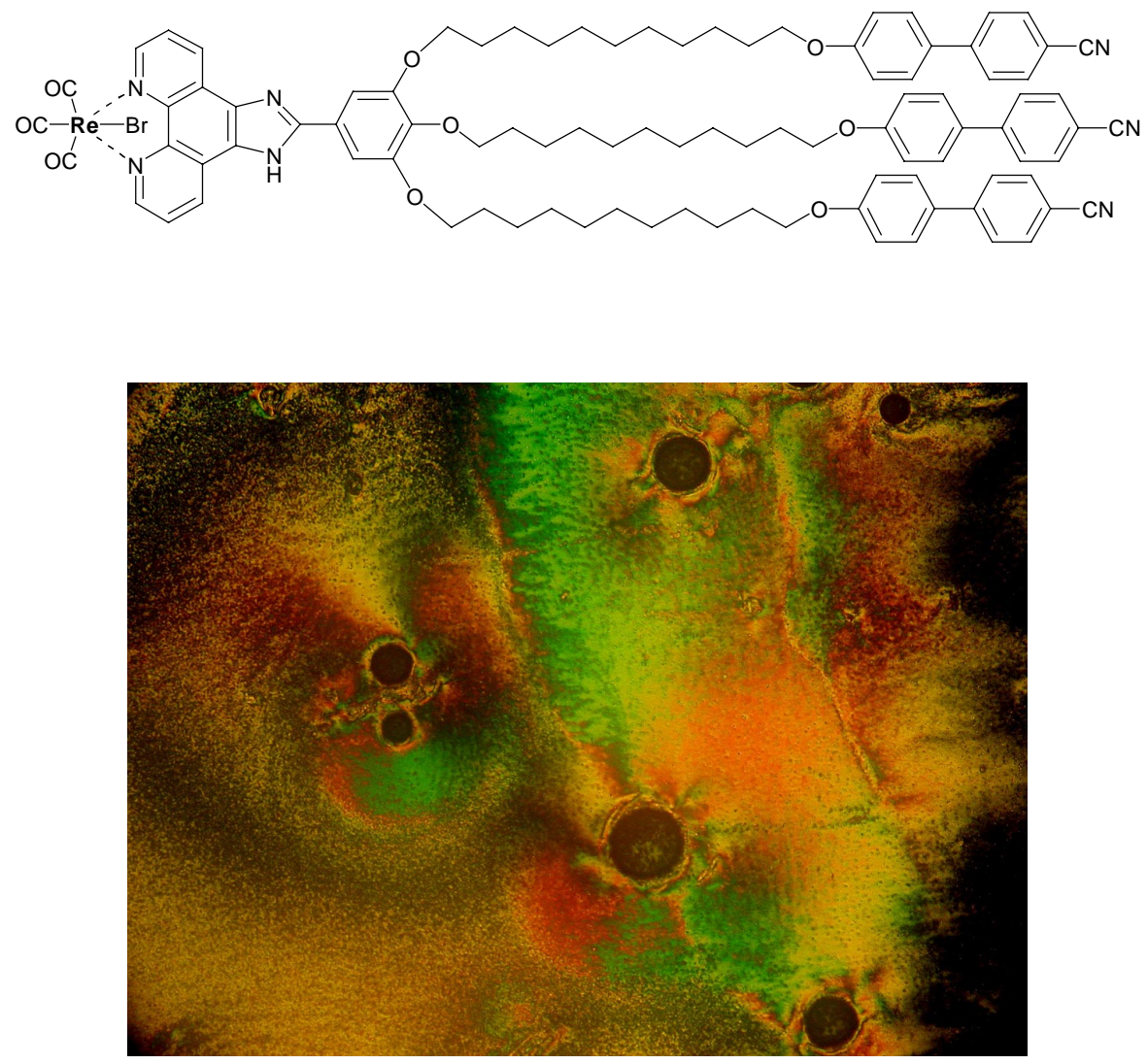

Optical texture of the $\mathrm{Col}_{\mathrm{r}} \mathrm{L}$ phase upon cooling at $90{ }^{\circ} \mathrm{C}$. 


\section{$\underline{\text { XRD diffractograms }}$}

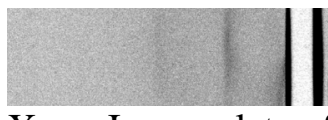

X-ray Image plate of the $\mathrm{SmA}_{\mathrm{Col}}$ phase of $\operatorname{Re}(\mathrm{CO})_{3} \mathrm{~L}^{3,4}$ at $60^{\circ} \mathrm{C} \cdot \mathrm{d}_{001}=45.5, \mathrm{~d}_{002}=22.6$, broad scattering $=4.5 \AA$.

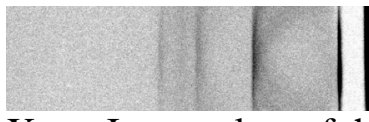

X-ray Image plate of the lamello-columnar phase of $\operatorname{Re}(\mathrm{CO})_{3} \mathrm{~L}^{3,4,5}$ at $80^{\circ} \mathrm{C} . \mathrm{d}_{2}=33.35, \mathrm{~d}_{3}=$ $20.75, \mathrm{~d}_{4}=18.75, \mathrm{~d}_{5}=16.45$.

X-ray pattern of the $\mathrm{SmA}_{\mathrm{Col}}$-type phase of $\mathbf{L}_{\mathrm{III}}$ at $80^{\circ} \mathrm{C}$. The following diffraction peaks are observed: $\mathrm{d}_{001}=50.0 \AA, \mathrm{d}_{002}=25.0 \AA, \mathrm{d}=14.0 \AA$, and the two broad scattering at $4.5 \AA$ and $3.5 \AA(\mathrm{h})$, respectively.

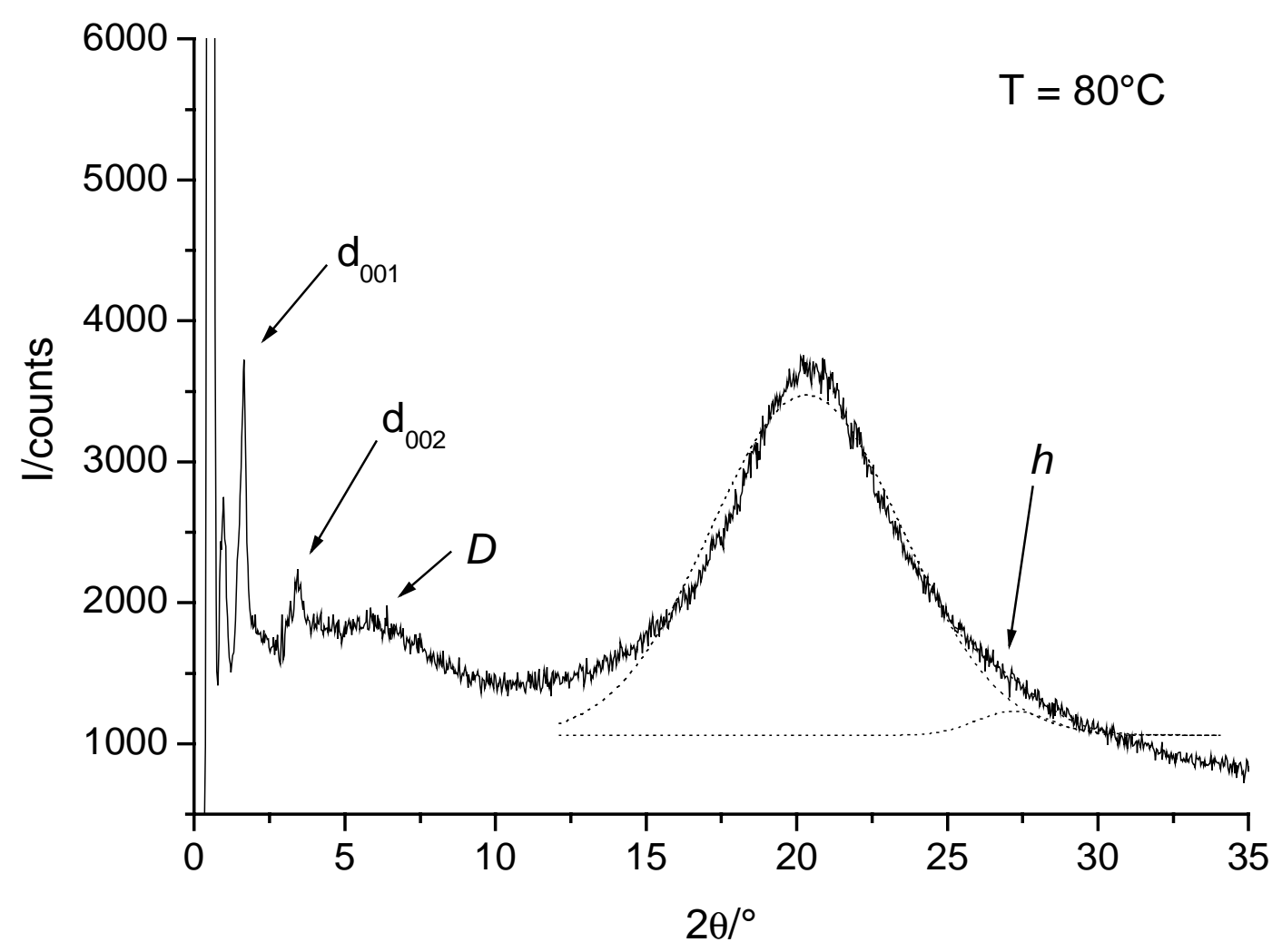




\section{Modelling}

Representation of the alternated packing of $\mathbf{L}_{\mathbf{I I I}}$ in the $\mathrm{SmA}_{\mathrm{Col}}$ phase built from two

snapshots of a single layer obtained by MD calculations. The columns forming ribbons can be seen from the alternated stacking of the phenanthroline macrocycles. The cyanobiphenyl mesogens are randomly tilted (see text).

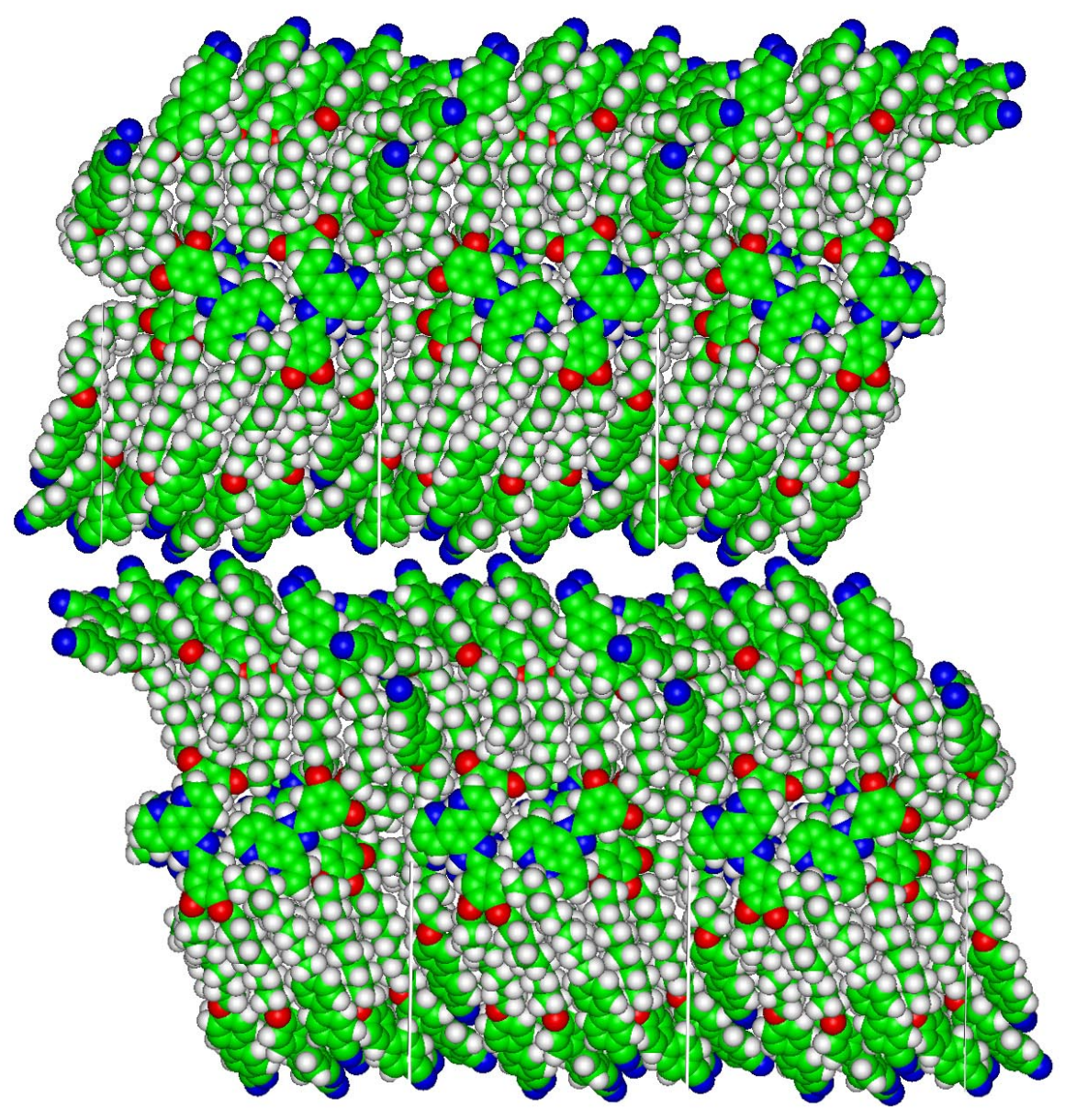


Snapshot of the packing of $\left[\operatorname{Re}(\mathrm{CO})_{3} \mathrm{Br} \mathbf{L}_{\mathrm{II}}\right]$ in the alternated $\mathrm{SmA}_{\mathrm{Col}}$ phase obtained from MD calculations. Short columns forming ribbons can be seen from the alternated stacking of the phenanthroline macrocycles. The cyanobiphenyl mesogens are randomly tilted (see text).

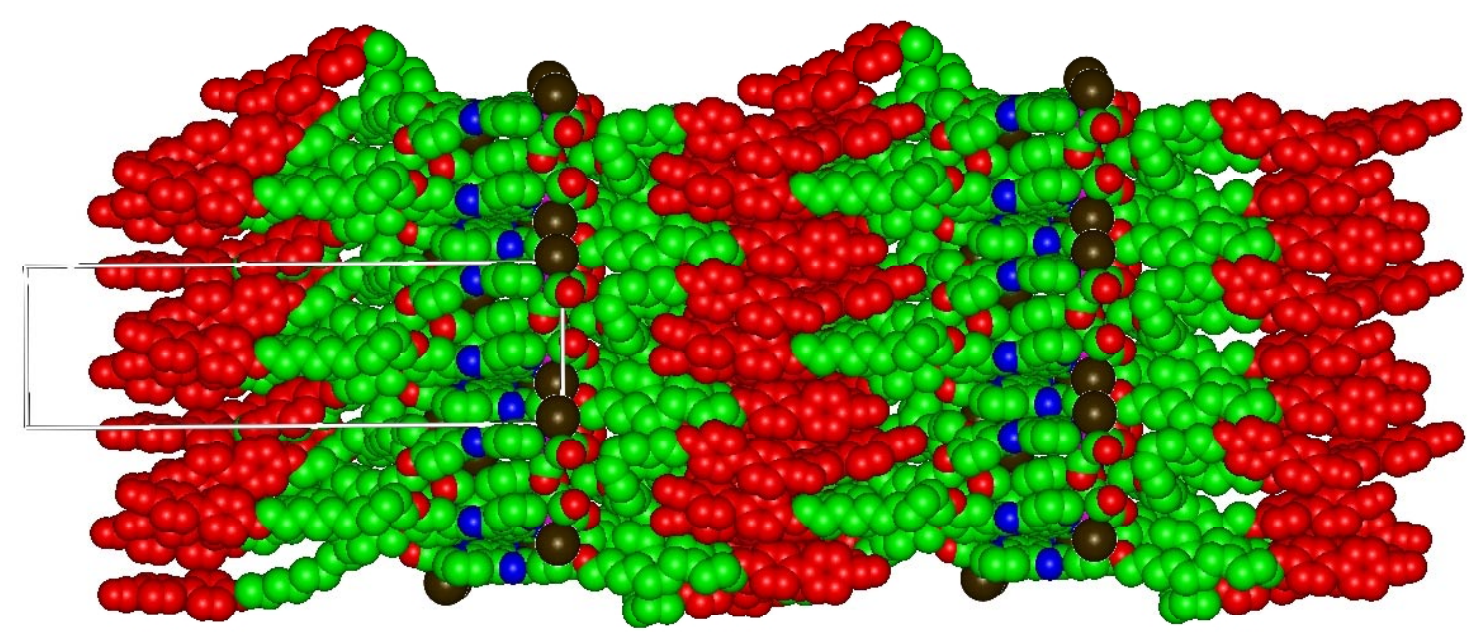




\section{Luminescence spectra}

Luminescence spectrum of $\left[\mathrm{Nd}(\mathrm{tta})_{3} \mathbf{L}_{\mathbf{I}}\right]$ at room temperature

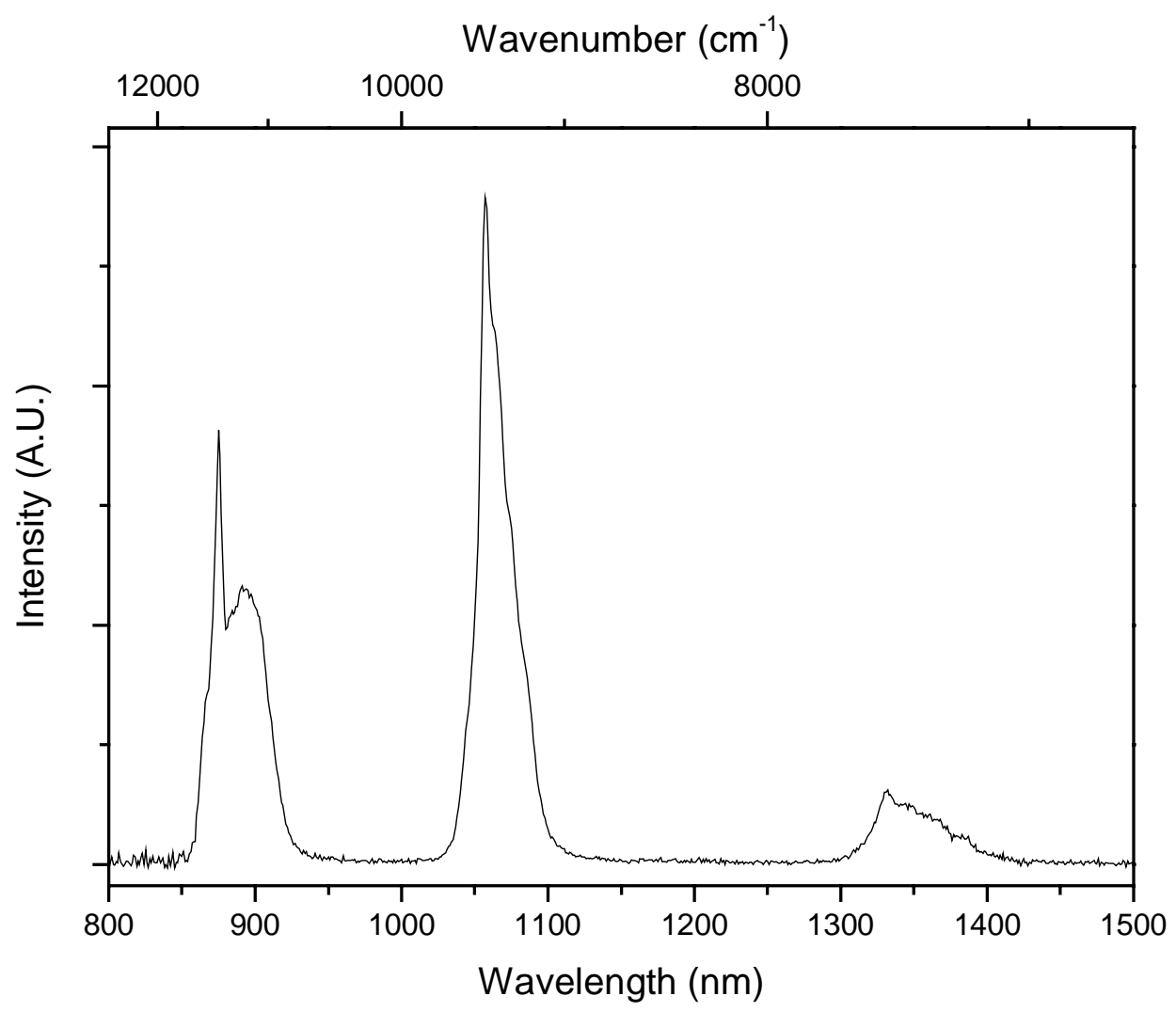


Luminescence spectrum of $\left[\mathrm{Sm}(\mathrm{tta})_{3} \mathbf{L}_{\mathbf{I I}}\right]$ at room temperature.

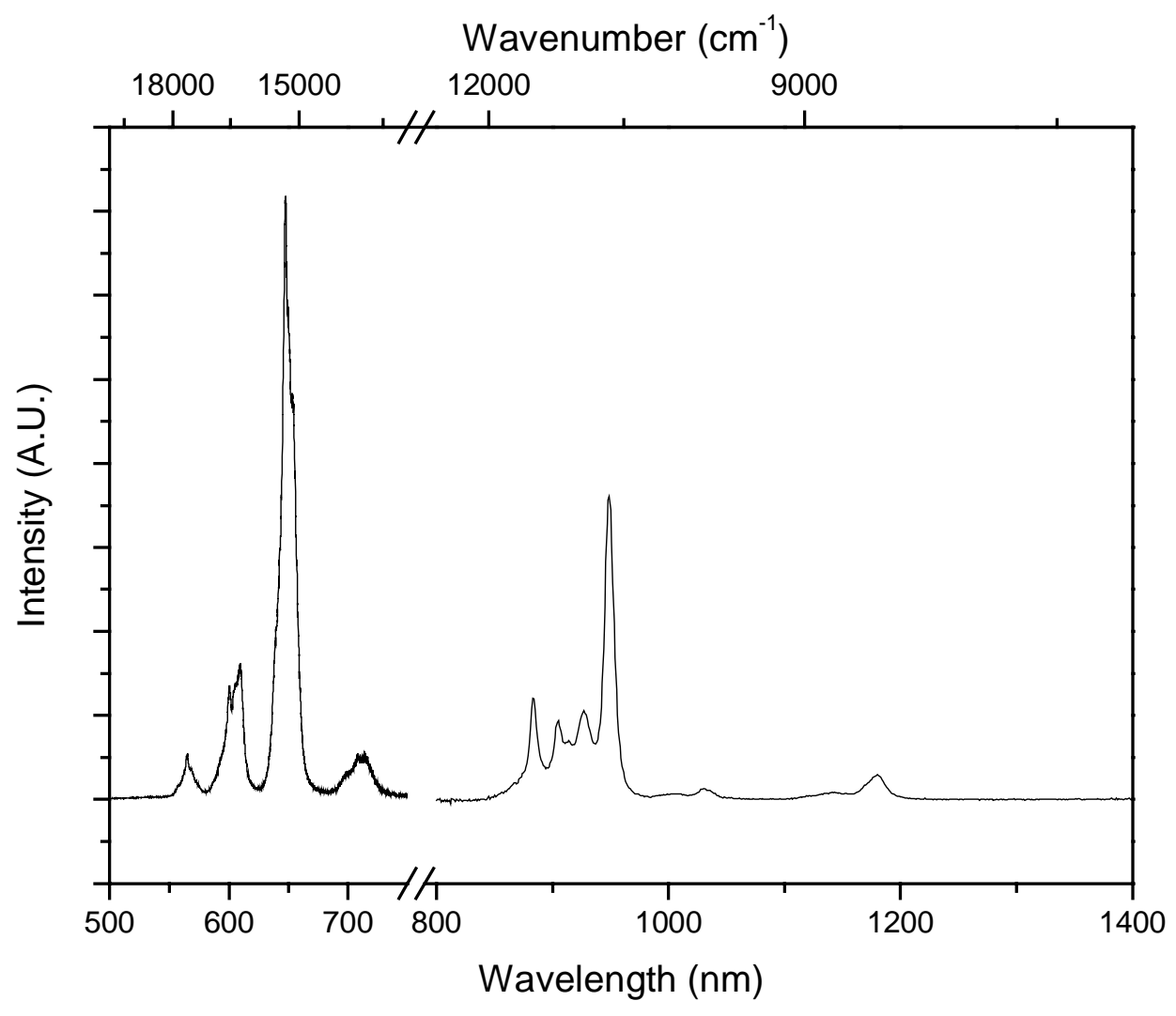


Luminescence spectrum of $\left[\mathrm{Eu}(\mathrm{tta})_{3} \mathbf{L}_{\mathrm{III}}\right]$ at room temperature.

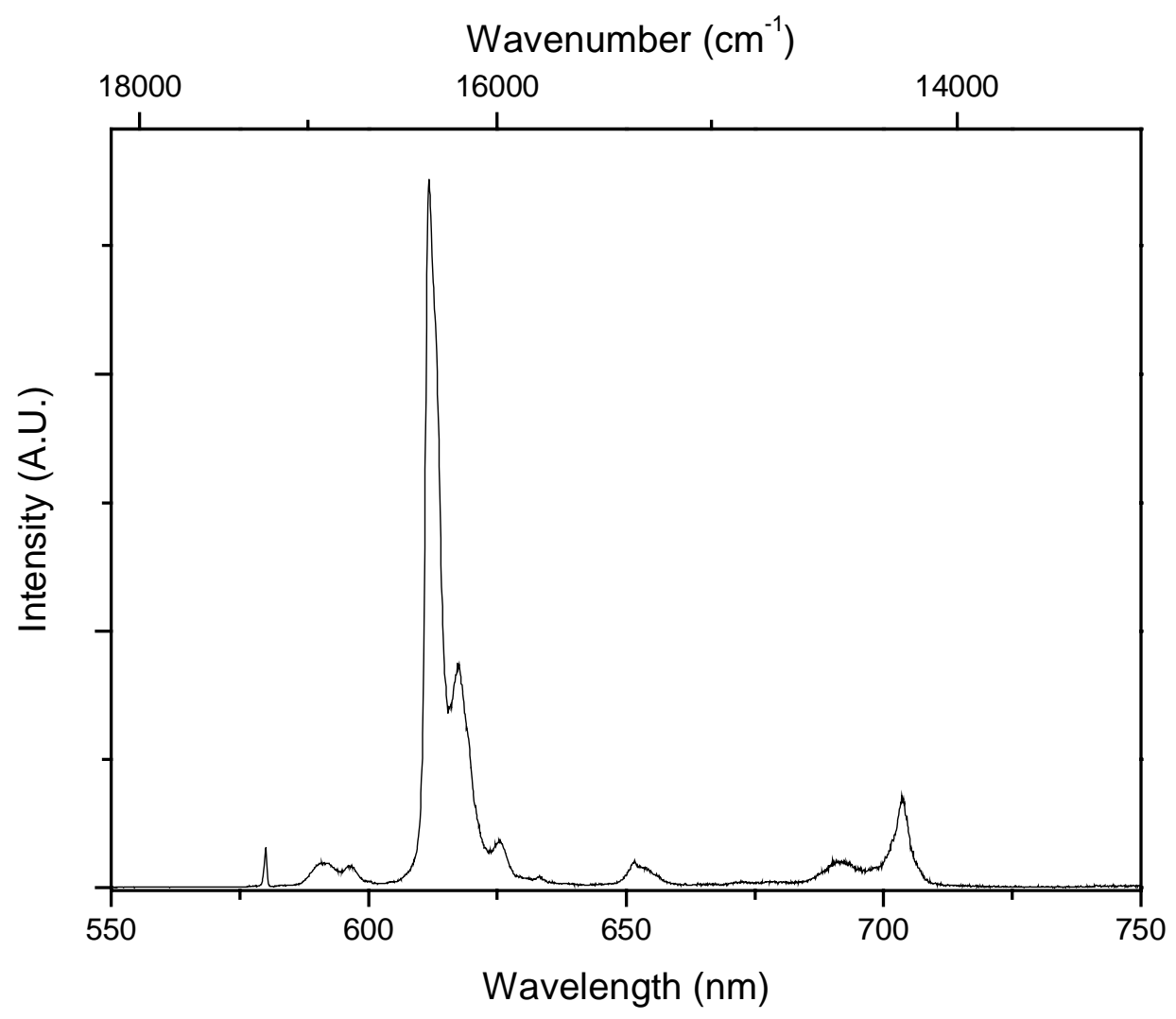


Luminescence spectrum of $\left[\operatorname{Er}(\operatorname{tta})_{3} \mathbf{L}_{\mathbf{I I}}\right]$ at room temperature.

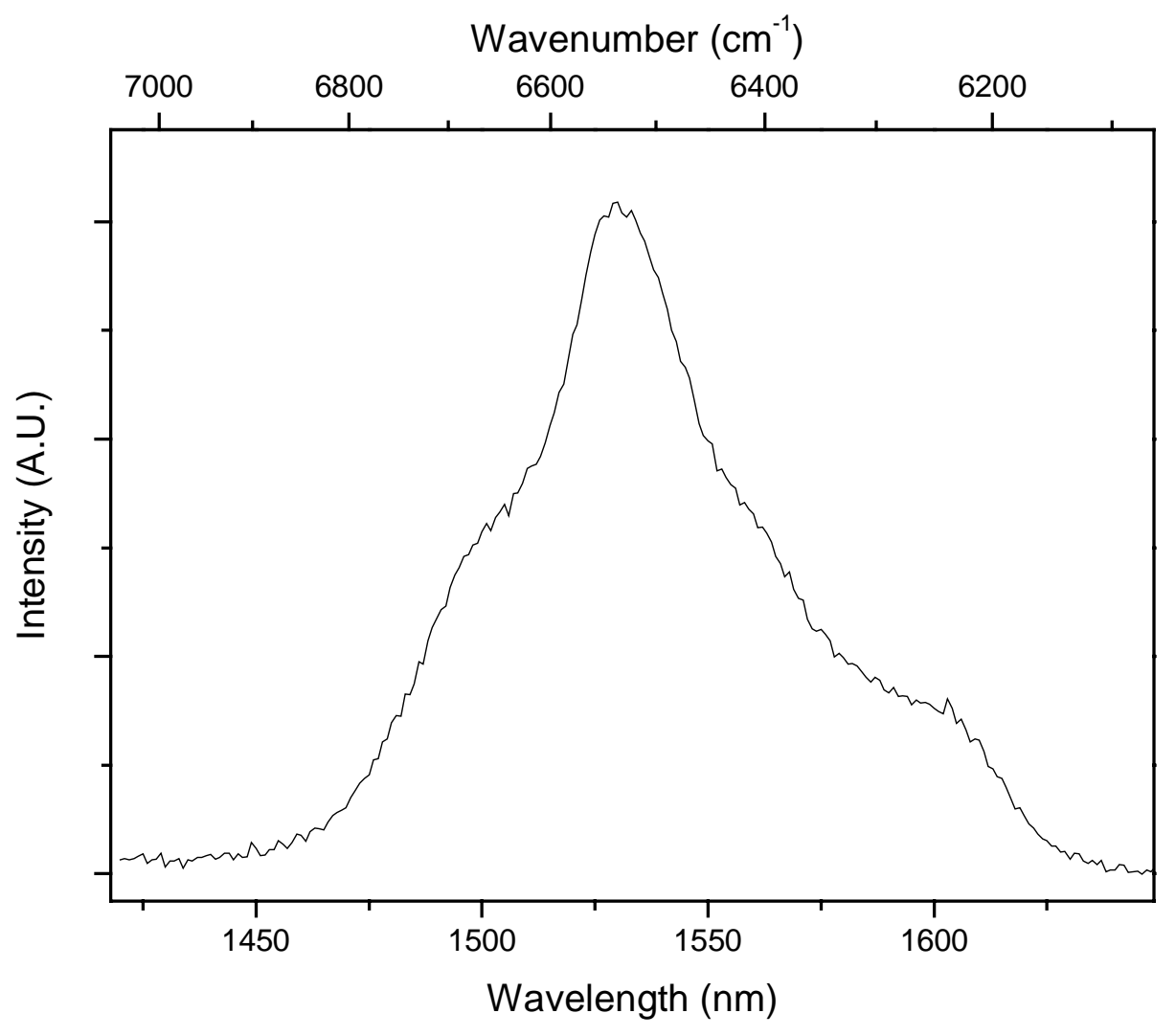


Luminescence spectrum of $\left[\mathrm{Yb}(\mathrm{tta})_{3} \mathbf{L}_{\mathrm{II}}\right]$ at room temperature.

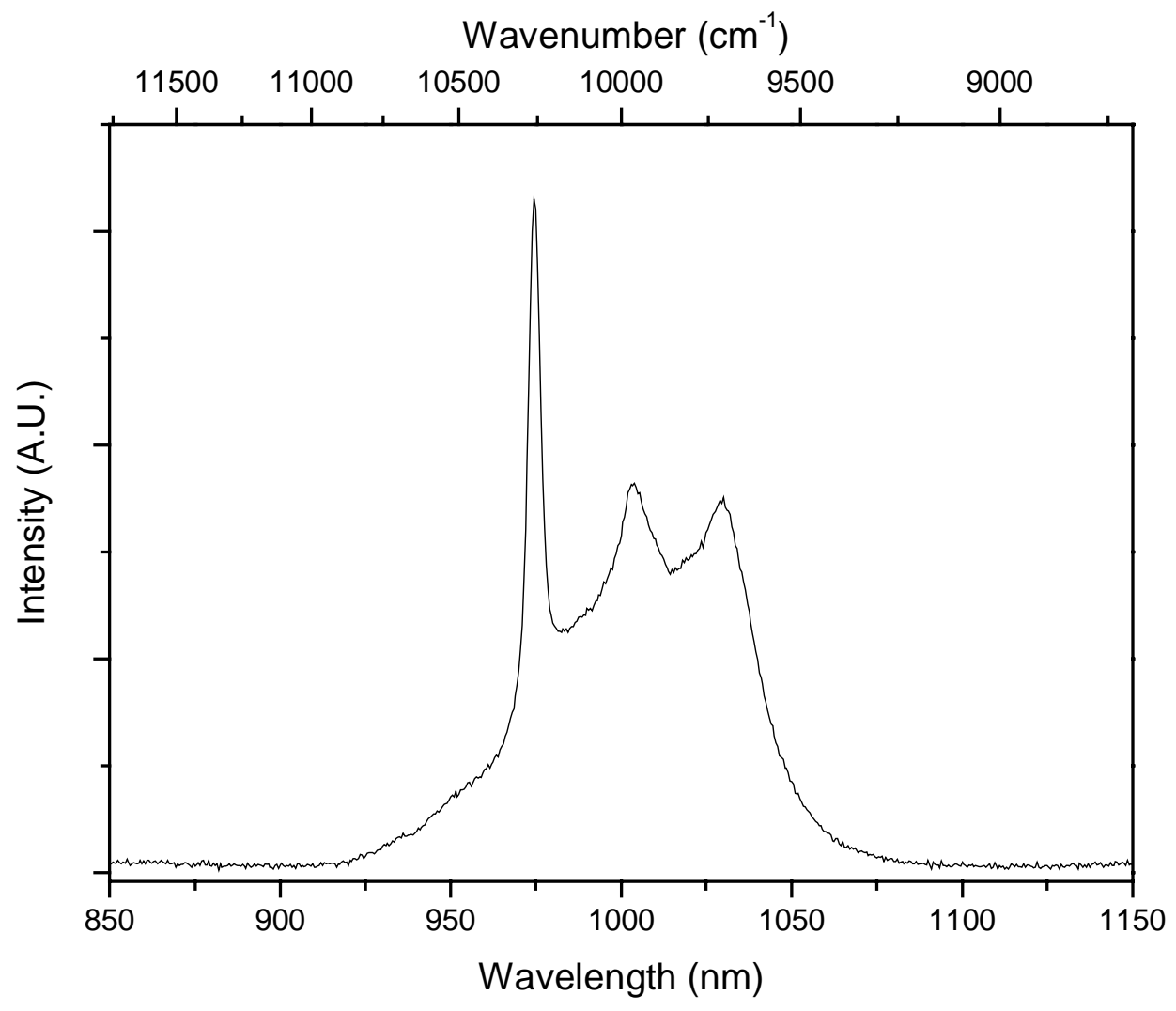

